Monte Carlo Calculations of the

Effective Area and Directional

Response of a Polyethylene-Moderated

Neutron Counter

R. C. Byrd

J. D. Drabanski*

B. L. Barraclough 


\section{DISCLAIMER}

This report was prepared as an account of work sponsored by an agency of the United States Government. Neither the United States Government nor any agency thereof, nor any of their employees, make any warranty, express or implied, or assumes any legal liability or responsibility for the accuracy, completeness, or usefulness of any information, apparatus, product, or process disclosed, or represents that its use would not infringe privately owned rights. Reference herein to any specific commercial product, process, or service by trade name, trademark, manufacturer, or otherwise does not necessarily constitute or imply its endorsement, recommendation, or favoring by the United States Government or any agency thereof. The views and opinions of authors expressed herein do not necessarily state or reflect those of the United States Government or any agency thereof. 


\section{DISCLAIMER}

Portions of this document may be illegible in electronic image products. Images are produced from the best available original document. 


\title{
MONTE CARLO CALCULATIONS OF THE EFFECTIVE AREA AND DIRECTIONAL RESPONSE OF A POLYETHYLENE-MODERATED NEUTRON COUNTER
}

\author{
by
}

\author{
R. C. Byrd, J. D. Drabanski, and B. L. Barraclough
}

\begin{abstract}
Two recent reports on Monte Carlo studies have examined the angular response of a multiple-rod neutron scintillator and the energy response of a moderated ${ }^{3} \mathrm{He}$ neutron counter. This report extends those studies to provide calculations of the effective area and angular sensitivity of a polyethylene-moderated neutron detector that has multiple ${ }^{3} \mathrm{He}$ tubes. The results (1) provide a more accurate and general determination of the sensor's detection efficiency, (2) suggest new techniques for obtaining information about the source direction, and (3) allow evaluation of proposals to improve the high-energy detection efficiency by using the production of $(n, 2 n)$ neutrons in high-density material added to the moderator.
\end{abstract}

\section{INTRODUCTION: EARLIER STUDIES}

Motivation. At Los Alamos National Laboratory (LANL), the original development of sensors for space-based neutron measurements dates back to the early $1960 \mathrm{~s}^{1}$ Their basic principle is similar to that of the Bonner spheres commonly used for neutron spectroscopy. ${ }^{2}$ Subsequent developments in computer codes for Monte Carlo simulation of neutron transport allow us to examine possible modifications that may improve the detector's sensitivity or provide directional information about sources of incident neutrons. This report combines previous calculations for a space neutron sensor ${ }^{3}$ based on moderated ${ }^{3} \mathrm{He}$ tubes with recent studies ${ }^{4-6}$ of multielement scintillation detectors. The complex detectors used in these studies lead to a redefinition of detection efficiency in terms of effective area, that is, the projected area of an equivalent detector with $100 \%$ efficiency. This approach makes it straightforward to extend the calculations of the directional response from the multi-rod scintillation detectors to the multi-tube ${ }^{3} \mathrm{He}$ counters. Comparisons on the basis of effective area also simplify the evaluation of modifications in the detector design, such as changes in size or moderator composition. Of particular interest are possible improvements in detection efficiency that have been reported for health-physics dosimeters designed for use at neutron energies above $10 \mathrm{MeV} \cdot 7,8$

Contents. Section 2 reviews the previous report on space-based neutron detectors and updates the calculations using the formalism of effective area instead of conventional detection efficiency. In Sec. 3 we extend this concept to measurements for fluxes with different angular orientations, and then we invert the process to determine the directional 
characteristics of the incident flux using the relative count rates for the different ${ }^{3} \mathrm{He}$ tubes. Section 4 describes the evaluation of possible improvements in detection efficiency provided by high-energy $(n, 2 n)$ reactions, and Sec. 5 presents a summary of our results.

\section{EFFECTIVE AREAS}

Overview. This section repeats and extends the previous analyses of detector efficiency ${ }^{3}$ using calculations of effective detector area ${ }^{6}$ instead of the more conventional detection probability per incident neutron. Essentially, detection efficiency is a quantity most suitable for controlled situations in which a uniform parallel beam of neutrons is incident on a well-defined projected area. Often, however, neither the detector geometry nor the beam orientation is well controlled; for example, neutrons can be incident from several different directions. In such cases it is more convenient to work in terms of the size of an equivalent detector that is perfectly efficient for all incident neutrons. At least conceptually, one can then visualize the instrument's response to different radiation fields without considering details of its geometry or operation.

Detector Model. Appendix A includes an input file describing the Los Alamos Neutron Detector (LAND) in terms of the LANL Monte Carlo N-Particle (MCNP) code, ${ }^{9}$ and Fig. 2.1 shows various sections through the MCNP geometry. The most important features of the detector are the substantial $(9.144-\mathrm{cm}$ radius) polyethylene moderator (material 1 in Fig. 2.1) and the six counter tubes. The figure also shows two different relative orientations that have been used for the tubes and the moderator. In the actual detector, the two larger tubes, which are high-sensitivity 4 -atm ${ }^{3} \mathrm{He}$ counters (material 2), are placed to the left and right inside the curved moderator surface. The alternative, "rotated" arrangement used in the previous calculations ${ }^{3}$ places these tubes at the top and bottom, next to the flat moderator surfaces that mate with the detector electronics. Whenever these different orientations are likely to significantly affect our conclusions, we will comment on the observed differences. The four smaller tubes are medium- and low-sensitivity ${ }^{10} \mathrm{BF}_{3} / \mathrm{Ar}$ counters (material 3), which are not emphasized in the present study. Also included with all figures are the $x-, y$-, and $z$-axes used throughout this report; the $x$-axis indicates the direction across the two large ${ }^{3} \mathrm{He}$ tubes, the $y$-axis is normal to the plane of the tubes, and the $z$-axis is oriented along the tube axes.

Previous Results. Most of the earlier calculations ${ }^{3}$ were formulated in terms of a detection efficiency defined as the probability that a neutron entering the detector will be moderated in the polyethylene and then captured in the ${ }^{3} \mathrm{He}$ gas, thereby producing a detectable output signal. Because this probability depends on the neutron's incident energy and angle, the response is described in terms of energy distributions such as those shown in Fig. 2.2a for the rotated moderator. As expected for a nearly symmetric detector, ${ }^{6}$ the efficiencies at high energies (above $100 \mathrm{keV}$ ) are essentially independent of the incident angular distribution. (The efficiencies for random directions in this figure are renormalized from those in Fig. 4.1 of Ref. 3. Because half of the randomly generated neutrons were directed away from the detector, the efficiencies plotted there were a factor of 2 low.) For the rotated moderator, the efficiency reaches a maximum value of $7 \%$ near $1 \mathrm{MeV}$; for the actual geometry this value is about $6 \%$. At lower energies the neutrons are absorbed before reaching the gas counters, and at higher energies they escape before being moderated to thermal energies. The random fluence provides a higher efficiency at low energies because some of the neutrons are incident almost directly onto the exposed ends of the gas counters.

Effective-Area Definitions. We now switch our formulation from detector efficiency $\epsilon$ to effective area $A_{\text {eff }}$. A problem with the standard definition of $A_{\text {eff }}$ as the product of detection probability and projected area $\left(A_{\text {eff }}=\epsilon A\right)$ is the difficulty of defining an area 

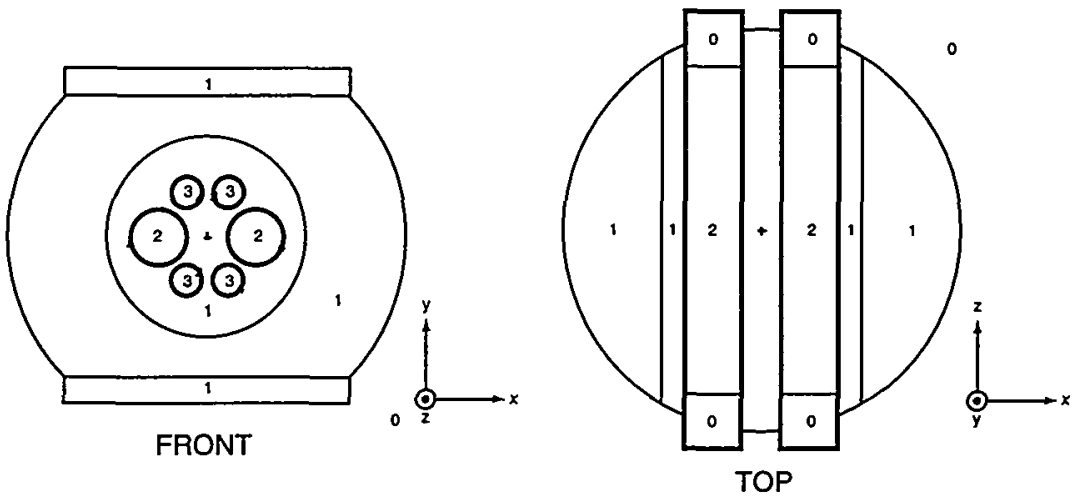

ACTUAL DETECTOR ORIENTATION

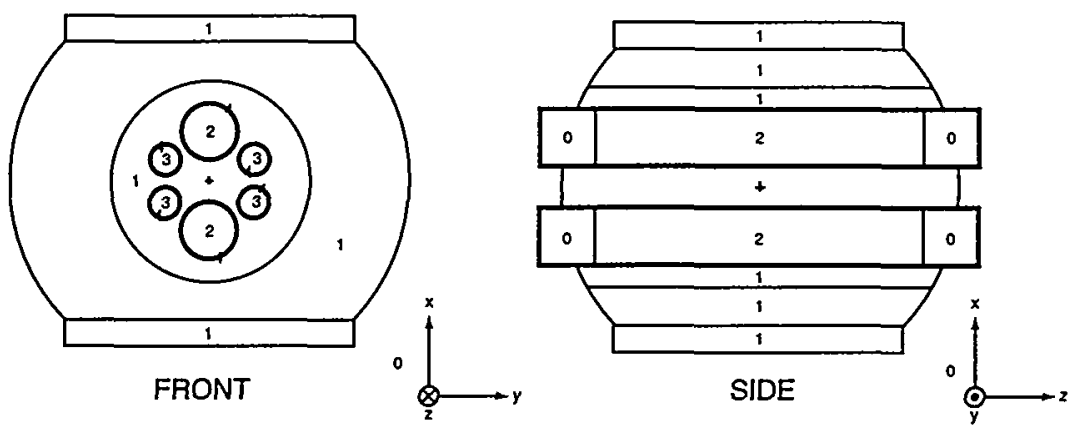

ROTATED DETECTOR ORIENTATION

Fig. 2.1. Computer model of the sensor used in the present calculations, showing the spherical moderator and the two large ${ }^{3} \mathrm{He}$ tubes. The MCNP model for the actual detector is shown in the upper panel, with the large tubes located inside the curved moderator surfaces. Previous calculations ${ }^{3}$ used a rotated moderator that placed the two ${ }^{3} \mathrm{He}$ tubes next to the flat surfaces, as shown in the lower panel. Both orientations are compared in the present work. The small axes indicate the reference directions.

for odd-shaped detectors (as for the four-rod scintillator in Ref. 6) or complex angular distributions (as for our random incident fluence). This problem was avoided in Ref. 6 by defining $A_{\text {eff }}$ not as efficiency times projected area, but as efficiency per unit fluence $\phi$ in neutrons $/ \mathrm{cm}^{2}$. In practice, $A_{\text {eff }}$ can be calculated simply by dividing the measured (or calculated) detection probability per incident neutron by the fluence $\phi$ at the detector location. For the trivial case of a single neutron incident normal to a well-defined area, the conventional result is obtained: $\phi=1 / A ; A_{\text {eff }}=\epsilon / \phi=\epsilon A$. More importantly, however, this procedure for calculating efficiency per unit fluence can be employed for any geometry, including the present case of a randomly directed flux.

Comparison with Present Calculations. To verify the consistency of the previous and present results, in Fig. 2.2b we first compare the area-efficiency products for the parallel-beam MCNP3B calculations from Fig. 2.2a (that is, from Ref. 3) with the corresponding MCNP4A values from this work. The calculations are labeled "estimated" because of an artifice used to obtain the efficiency for our detector, whose nominal radius of $9.144 \mathrm{~cm}$ is truncated to $\pm 7.62 \mathrm{~cm}$ in the $\pm x$ directions because of the two mounting plates. The calculation therefore employed a radial distribution that extended only to $7.62 \mathrm{~cm}$; to compensate partially for this truncation, the distribution was weighted as $r^{2}$, not as $r$, to 


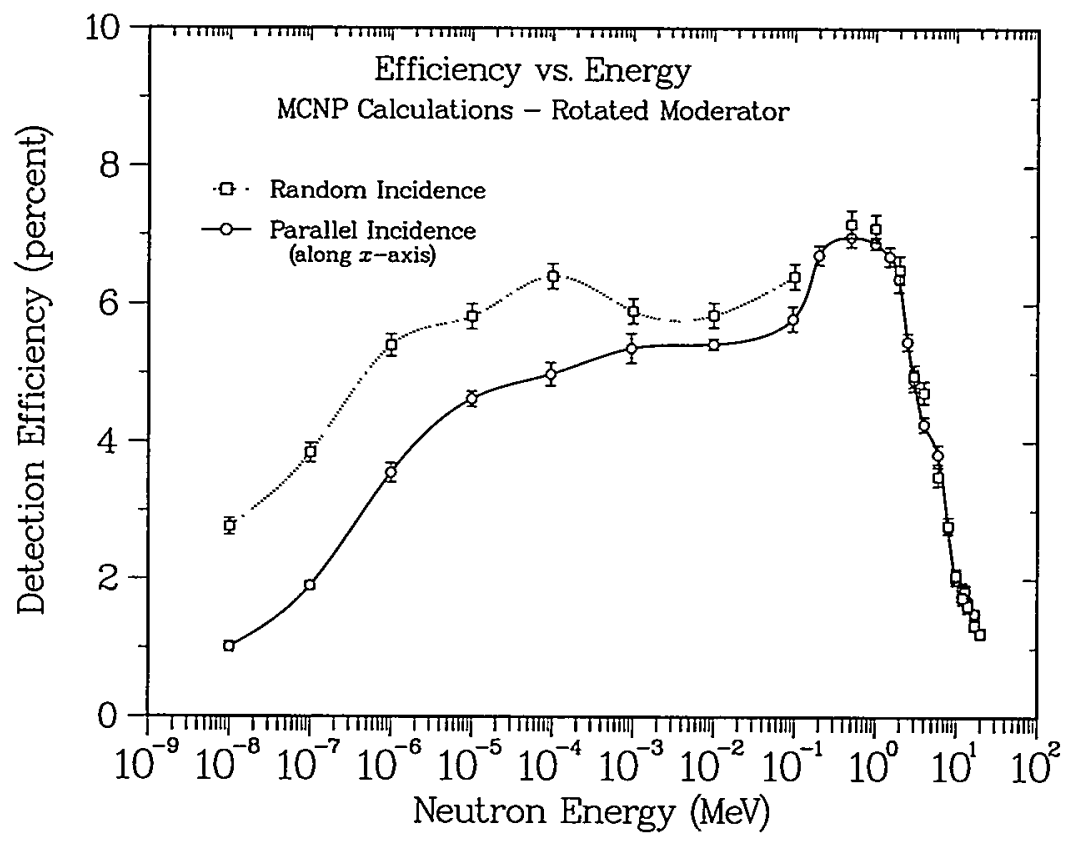

Fig. 2.2a. MCNP calculations of the rotated sensor's detection efficiency below $20 \mathrm{MeV}$. Two different incident angular distributions, random and parallel to the $x$-axis, are shown.

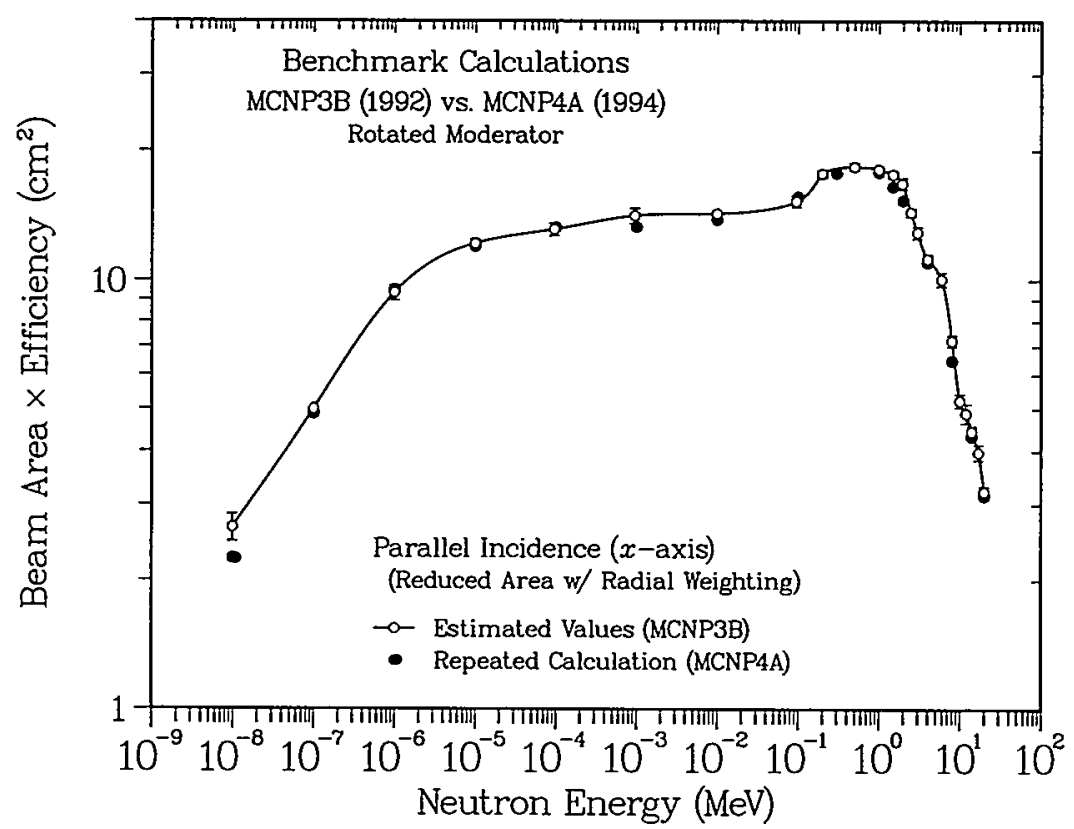

Fig. 2.2b. Comparison between the effective areas calculated using MCNP3B (see Ref. 3) and the corresponding areas using MCNP4A. Both calculations use a modified radial distribution to compensate for limitations imposed by the definition of effective area in terms of projected detector area. 
increase the number of neutrons striking near the edge of the detector. As a first test, the new calculations repeated this procedure using MCNP4A and obtained the statistically equivalent results shown in Fig. 2.2b.

Effective-Area Comparisons. By calculating the effective area as the efficiency per unit fluence, we can now check the accuracy of the previous approximate calculations and replace their results with those from the exact approach. Figure 2.2c compares the old and new effective-area calculations for the rotated moderator. Although the overall agreement is quite good, the approximation overestimates the true area by about $13 \%$ near the peak at $1 \mathrm{MeV}$. The agreement is sufficient, however, that the resulting conclusions in Ref. 3 about the characteristics of the space-based neutron detector should still be valid.

Size Variations. An excellent example of the viewpoint encouraged by the effectivearea formulation concerns the effect of variations in the dimensions of the detector. In the earlier study (see Ref. 3, Sec. 7), instead of laboriously changing the sizes of the incident beam and each of the 50-odd individual detector dimensions, we simply varied the densities of the four different materials-upward by a factor of 1.2 and downward by factors of 0.8 and 0.6 - and then examined the resulting changes in detection efficiency. Although this approach correctly reflects the changes in neutron transport, it certainly does not represent a workable proposal for an actual detector. To test this approximation, in the present work we have made calculations that either decrease the actual dimensions of the moderator from $9.144 \mathrm{~cm}$ to $8.66 \mathrm{~cm}$ or that reduce the polyethylene density by the same fraction (about $5 \%$ ). All other detector components (tubes, gas, etc.) were kept the same. Figures 2.3a,b examine the effect of these two changes - density versus dimensions-on the energy-dependent effective areas. First, Fig. 2.3a compares the effect of reducing the polyethylene density versus reducing the polyethylene dimensions. Although the results are in general agreement, it is clear near $1 \mathrm{MeV}$ that obtaining precise values for a real

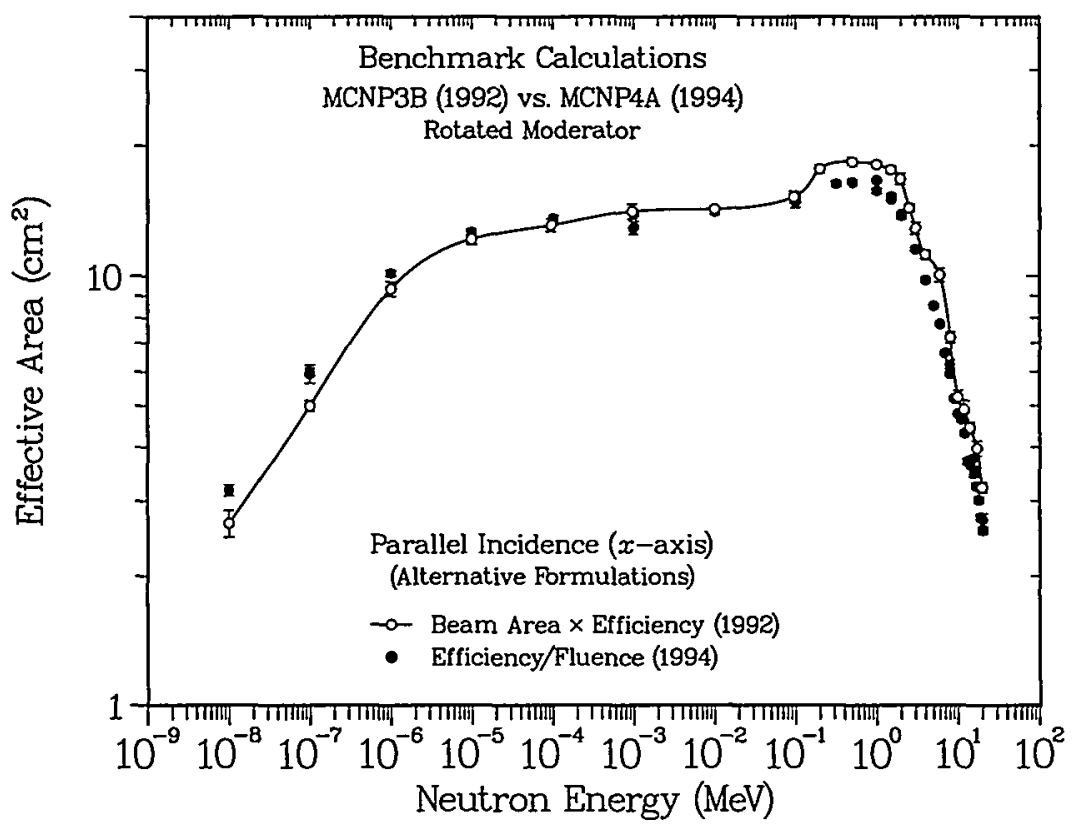

Fig. 2.2c. Comparison between approximate (Fig. 2.2b) and exact calculations of the effective detector area for neutrons at parallel incidence along the rotated moderator's $x$-axis (across the ${ }^{3} \mathrm{He}$ tubes). 


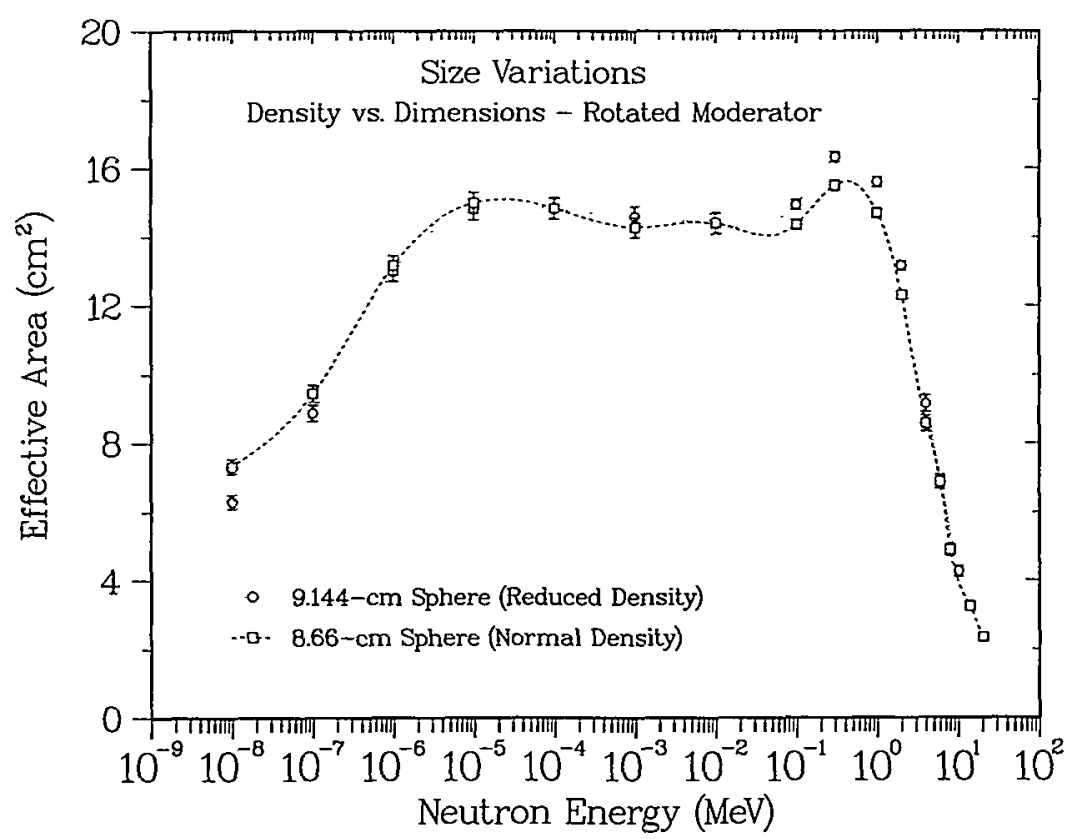

(a)

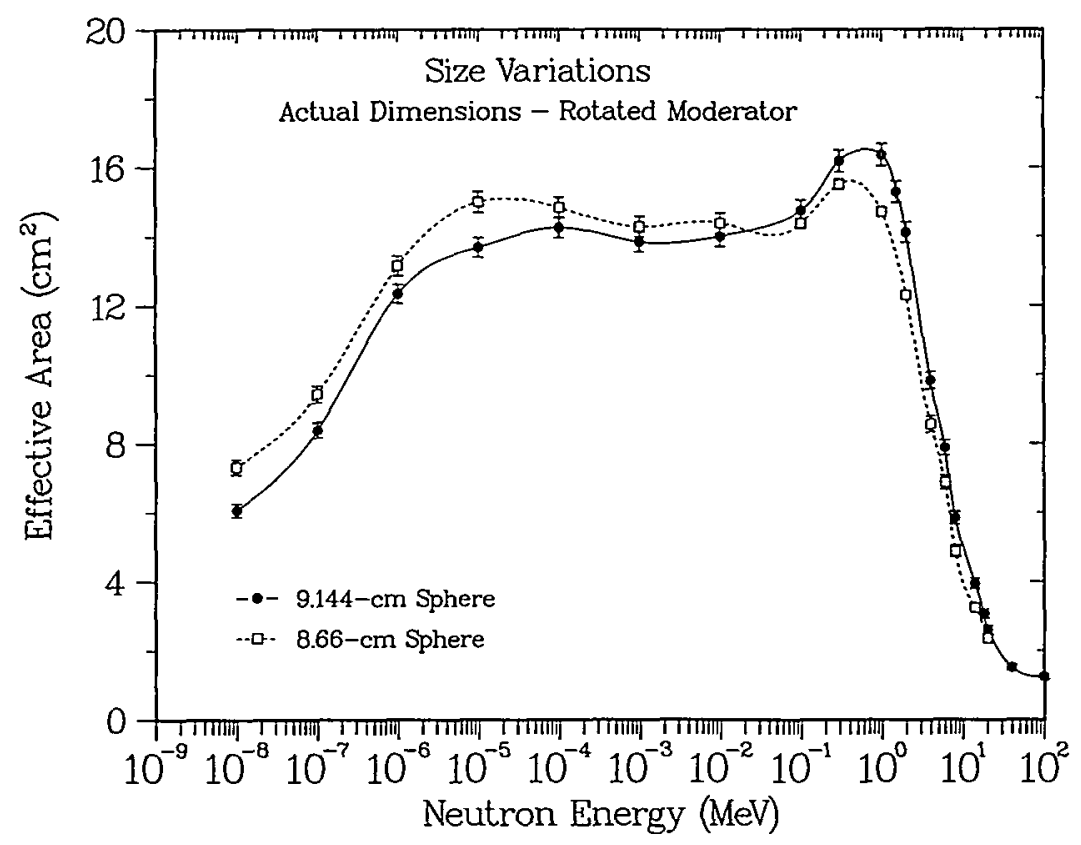

(b)

Figs. 2.3a,b. Effect on the effective area of decreasing the size of the (rotated) moderator by about $5 \%$. Part a compares the effect of simply changing the moderator density with that for actually reducing its size; part b compares the effective areas of the standard and reduced-size detectors using only changes in the polyethylene dimensions. 
detector requires changing the dimensions, not simply the densities. Qualitatively, these changes are about one-half those produced by the $20 \%$ decrease in overall density used in Ref. 3. Accordingly, Fig. 2.3b compares the effective areas for the large and small detectors, using only changes in the polyethylene dimensions. As in the approximate analyses shown in Fig. 7.2 of Ref. 3, the decrease in size raises the low-energy efficiency (by $10 \%$ at $1 \mathrm{eV}$ ) and lowers the high-energy efficiency (by $20 \%$ at $14 \mathrm{MeV}$ ), primarily because of changes in the balance between neutron attenuation and moderation.

Angular Distributions and Orientations. Thus far, the present calculations have emphasized the $x$-axis orientation for the rotated moderator used in Ref. 3 , that is, a parallel beam across the two large ${ }^{3} \mathrm{He}$ tubes, which are located near the two flat sides of the moderator. We now compare these parallel-beam calculations with those for randomly directed neutrons, using effective areas instead of the approximate efficiencies of Fig. 2.2. Figure 2.4 shows the results, which agree well with the earlier efficiencies when multiplied by the projected area of $263 \mathrm{~cm}^{2}$; for example, the $6.5 \%$ maximum efficiency for random incidence in Fig. 2.2a translates to the maximum $A_{\text {eff }}$ value of $17 \mathrm{~cm}^{2}$ in Fig. 2.4. Again, the major reason for the difference between the parallel and random distributions at low energies is associated with direct leakage into the exposed ends of the ${ }^{3} \mathrm{He}$ tubes. This interpretation is supported by the results for the three orthogonal detector orientations shown in Fig. 2.5a. The $z$-axis values, for parallel incidence along the tube axes, show a pronounced low-energy increase. The $y$-axis values, for incidence normal to the plane defined by the two tubes, decrease at low energies because of the significant attenuation through the thick curved surface of the rotated moderator (see Fig. 2.1). Because the neutron mean free path increases with increasing energy, above about $4 \mathrm{MeV}$ the three responses become almost identical, as emphasized by the enlarged view of the high-energy region shown in Fig. $2.5 \mathrm{~b}$.

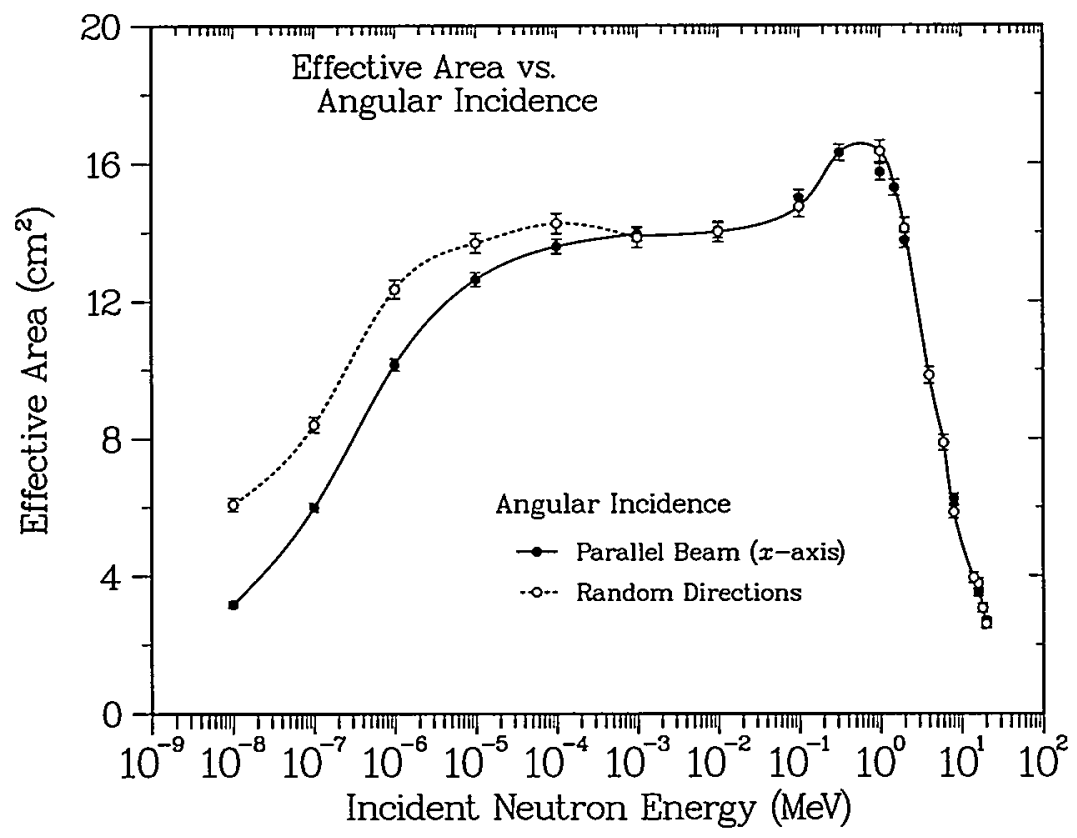

Fig. 2.4. Comparison between effective areas for random incidence and incidence parallel to the $x$-axis. The low-energy enhancement in the random case is caused by leakage into the exposed ends of the ${ }^{3} \mathrm{He}$ tubes. 


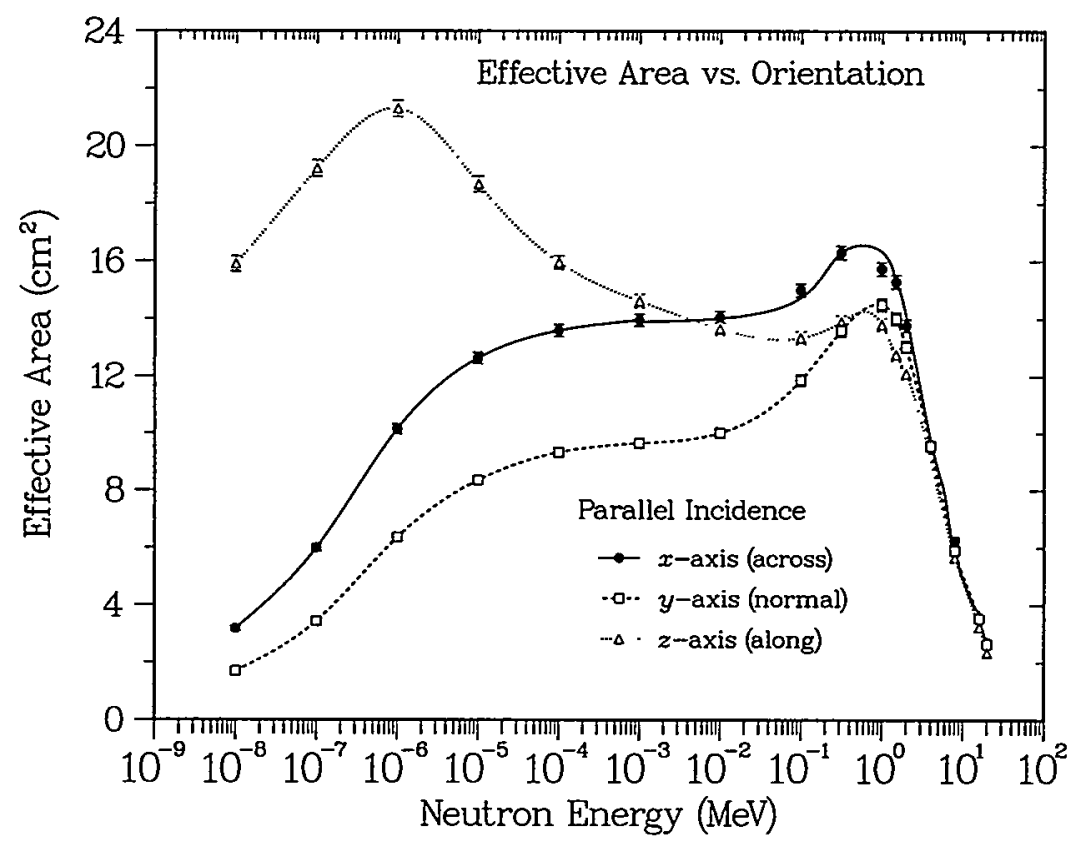

(a)

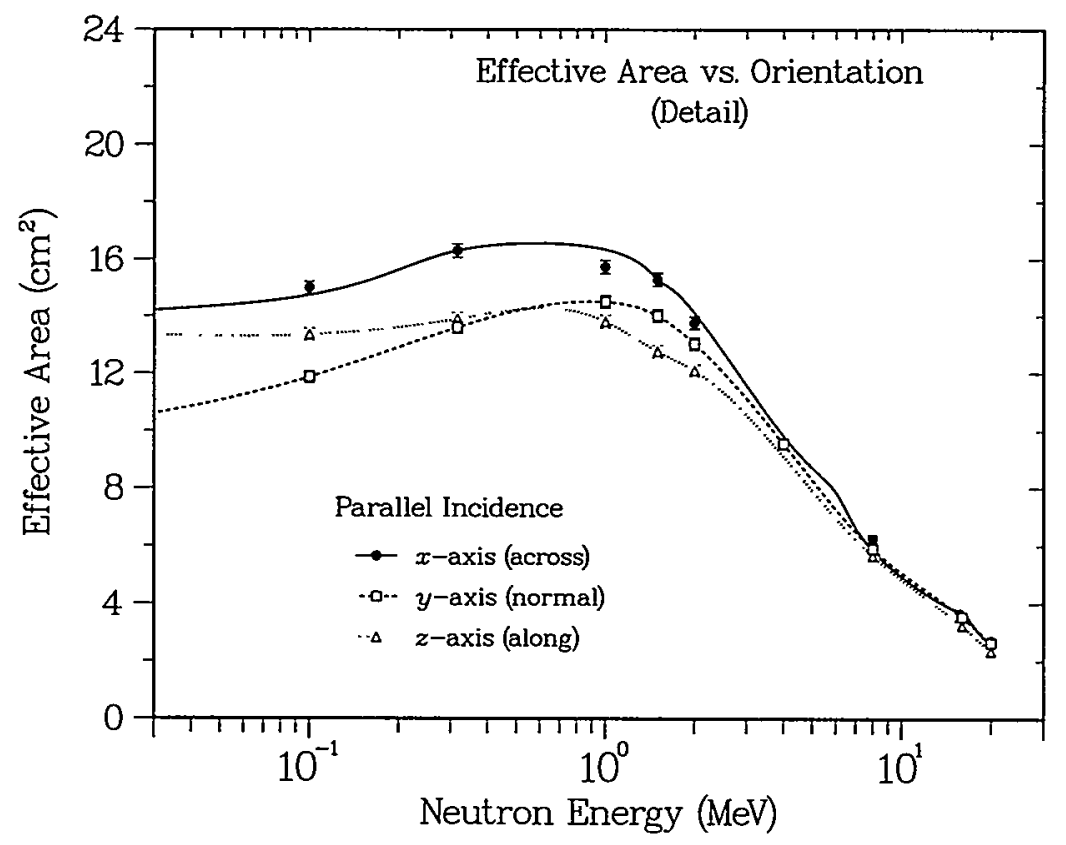

(b)

Figs. 2.5a,b. Effective areas for parallel incidence along the $x^{-}, y$-, and $z$-axes. Part a shows the complete energy dependence; part $b$ focuses on the highest energies. The differences in low-energy response are discussed in the text. 


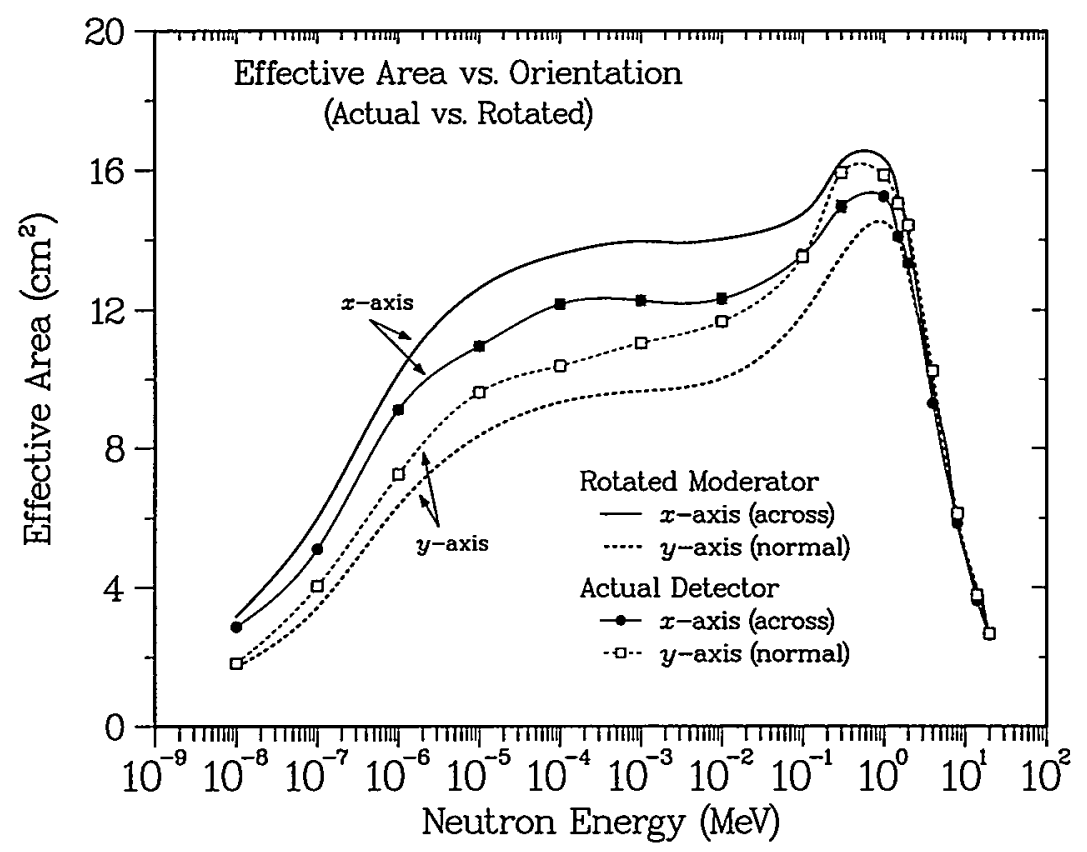

Fig. 2.6. Effective areas for parallel incidence along the $x$ - and $y$-axes, comparing results for the rotated moderator of Fig. $2.5 \mathrm{a}$ with the corresponding values for the actual detector.

Actual Detector. We close this section by including the analogous calculations to those in Fig. 2.5a, but for the actual detector shown in Fig. 2.1, which has the large ${ }^{3} \mathrm{He}$ tubes placed inside the rounded moderator surfaces instead of the flat ones. This rotation increases the $x$-axis shielding at the tube positions and decreases it along the $\pm y$-axis; as shown in Fig. 2.6, these changes bring the $x$ and $y$ curves closer together across the entire low-energy region and provide a more uniform angular response. The numerical values for the plot are tabulated in Appendix B.

Summary. Much of this section has been devoted to reproducing earlier ${ }^{3}$ benchmark efficiencies $\epsilon$ and converting the results to the new formulation of effective areas $A_{\text {eff. }}$. Accordingly, Figs. 2.2-2.4 replace the earlier approximate efficiency calculations with the more straightforward and accurate $A_{\text {eff }}$ approach developed for handling complex scintillator geometries. ${ }^{6}$ For the present sensor, the complications come not so much from the detector geometry as from the variations in incident angular distributions, such as for the case of random directions or parallel incidence along different axes (Figs. 2.4-2.6). The different orientations reveal that the detector's response varies significantly with direction for neutron energies below $1 \mathrm{MeV}$, either because of direct leakage into the exposed ends of the ${ }^{3} \mathrm{He}$ counters (at very low energies) or variations in the neutron attenuation in the moderating material (at somewhat higher energies). At energies above $1 \mathrm{MeV}$, however, the angular response becomes fairly uniform, especially for the geometry of the actual detector. The onset of this angular uniformity coincides with the peaking in the detector's detection efficiency, which is reached when the polyethylene thickness is sufficient to moderate the neutrons to thermal energies as they reach the locations of ${ }^{3} \mathrm{He}$ tubes. At higher energies the insufficient moderation for detectors of practical size reduces both the efficiency and the orientation differences. Finally, the comparison of calculations for the actual and rotated moderators demonstrates the detector's sensitivity to the exact location of the tubes. Changes in position, orientation, or separation can have surprisingly large effects on the response. 


\section{DIRECTIONALITY}

Overview. The large differences in the low-energy count rates for ${ }^{3} \mathrm{He}$ tubes at different locations provide a signature of a directional incident neutron flux. This interpretation of directionality is similar to one developed previously to separate the characteristics of the detector from those of the radiation field..$^{5}$ In this view the detector is an analyzer whose count-rate asymmetry reflects the polarization of the surrounding radiation field. Thus, instead of treating the detector as simply responding to the radiation field, we can use it as an instrument that determines the characteristics of this field-a polarimeter. This approach is followed in this section, which adapts the polarization discussions for segmented scintillators ${ }^{4,5}$ to the case of multi-tube moderated ${ }^{3} \mathrm{He}$ detectors.

Analyzing Power and Polarization. The detector's analyzing power $A$, which measures its ability to indicate the polarization $P$ of the incident beam, is defined for the $x$-axis in terms of the measured asymmetry $R_{x}=P_{x} A_{x}$ in the counting rates $\left(N_{+}, N_{-}\right)$for the two tubes lying in the $y-z$ plane:

$$
\begin{aligned}
& R_{x}=\left(r_{x}-1\right) /\left(r_{x}+1\right) \\
& d R_{x}=2 /\left(r_{x}+1\right)^{2} d r
\end{aligned}
$$

where

$$
\begin{gathered}
r_{x}=N_{+} / N_{-} \\
d r=r\left[\left(\frac{d N_{+}}{N_{+}}\right)^{2}+\left(\frac{d N_{-}}{N_{-}}\right)^{2}\right]^{1 / 2}=r\left(\frac{1}{N_{+}}+\frac{1}{N_{-}}\right)^{1 / 2} .
\end{gathered}
$$

The error analysis assumes that the statistical uncertainty is given by the square root of the number of counts. To illustrate the results, if a parallel beam is incident on the rotated moderator from the $-x$ direction, then it has $P_{x}=-1$ and $P_{y}=P_{z}=0$. For 50,000 neutrons at $1 \mathrm{MeV}$, such a beam gives $N_{-}=2936$ captures (for the closer tube at $x=$ $-2.0 \mathrm{~cm}$ ) and $N_{+}=1783$ captures (for the farther tube at $x=+2.0 \mathrm{~cm}$ ). These numbers give $R_{x} \pm d R_{x}=-0.251 \pm 0.014$; that is, the detector's analyzing power $A_{x}=R_{x} / P_{x}$ is 0.251 at $1 \mathrm{MeV}$. Similar calculations for other energies and orientations allow us to map out the complete energy-dependent functions $A_{x}, A_{y}$, and $A_{z}$ shown in Fig. 3.1. Because of the detector's symmetry, only $A_{x}$ is nonzero; its value drops rapidly above $0.1 \mathrm{MeV}$ because the mean free path inside the detector $(4.9 \mathrm{~cm}$ at $10 \mathrm{MeV})$ becomes comparable to the separation between the tubes. The figure also shows $A_{x}$ values for the actual detector geometry as open circles connected by the solid curve. These results are little changed from those for the rotated moderator, and their numerical values are tabulated in Appendix $B$. No corresponding $A_{y}$ and $A_{z}$ values are tabulated because they are trivially equal to zero in both geometries. Finally, the single measurement (the $\times$ symbol) was obtained using the actual detector and a plutonium/beryllium $(\mathrm{Pu} / \mathrm{Be})$ source at a LANL calibration facility. ${ }^{10}$ This point is plotted at the measured average energy of the neutron beam $(2.28 \mathrm{MeV}) ;^{11}$ because of scattering, this energy is below that $(\sim 4 \mathrm{MeV})$ expected for the $\mathrm{Pu} / \mathrm{Be}$ source by itself. The agreement with the calculations is unquestionably quite good.

Two-Tube Angle Determinations. Because zero analyzing power results in zero asymmetry regardless of the beam direction, the existing two-tube detector can only indicate directions about one axis and only at energies below a few $\mathrm{MeV}$. Figure 3.2a suggests a possible direction-indicating algorithm based on the measured polarization $P_{x}=P \cos \theta_{x}$ $=R_{x} / A_{x}$, where $A_{x}$ is the $x$-axis analyzing power shown in Fig. 3.1. For these calculations the beam axis was rotated around the detector in the $y$ - $z$ plane in $10^{\circ}$ steps from $0^{\circ}$ to $90^{\circ}$. Except near $45^{\circ}$, where a nearby $\mathrm{BF}_{3}$ tube probably interferes slightly with the neutron fluence inside the detector, it appears that the simple relation $\theta_{x}=\cos ^{-1} P_{x}$ gives excellent 


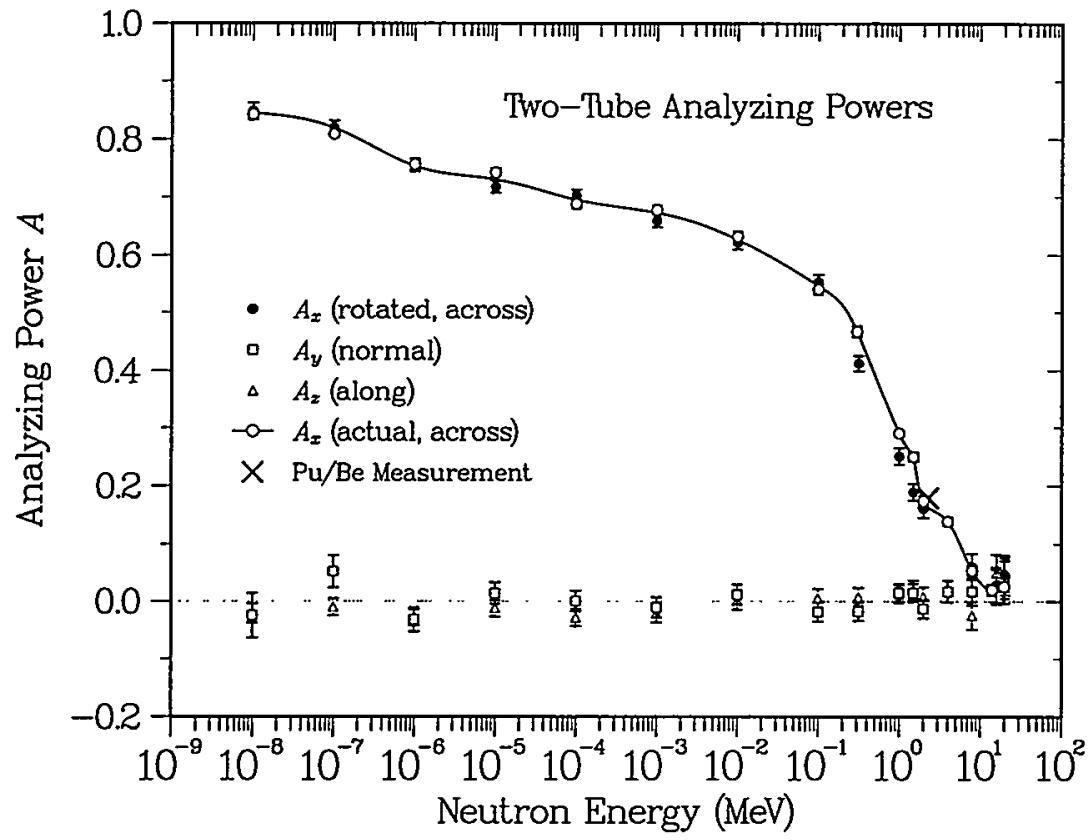

Fig. 3.1. Detector analyzing powers. For parallel beams along the axes of the two tubes (the $z$-axis) or normal to their plane (the $y$-axis), there can be no asymmetry in the count rate and hence no analyzing power. The neutron attenuation along the $x$-axis reduces the neutron flux so that the closer tube has a greater count rate, at least at low energies. Both detector orientations show similar behavior. The single measurement was obtained with a $\mathrm{Pu} / \mathrm{Be}$ calibration source.

angle estimates. The slight increase in the statistical uncertainties at $1 \mathrm{MeV}$ becomes even more pronounced at higher energies, as shown dramatically for $E_{n}=10 \mathrm{MeV}$ in Fig. 3.2b. Here it is clear that the near-zero analyzing power almost completely eliminates any accurate angle determination. If practical, using a larger moderator would allow the technique to be extended to higher energies.

Energy Dependence. Implicit in the discussion of Figs. 3.2a, $\mathrm{b}$ is a significant limitation in the applicability of the two-tube angle measurement. Because determining the polarization $P_{x}=R_{x} / A_{x}$ requires selecting the appropriate energy-dependent $A_{x}$ value, the incident neutron energy must either be known or measurable in some manner. The most common situation where this information is available is the case of time-of-flight (TOF) measurements, where the neutrons are produced in a single burst (at $t=t_{0}$ ) and their energies $E_{n}$ can be determined from their transit times $\left(t_{n}-t_{0}\right)$ over a distance $d$ as

$$
E_{n}=\frac{1}{2} M_{n} v_{n}^{2}=\frac{1}{2} M_{n} \frac{d}{\left(t_{n}-t_{0}\right)^{2}}
$$

where $M_{n}$ and $v_{n}$ are the neutron mass and velocity in the appropriate units.

Four-Tube Angles. Because TOF measurements are possible only in special situations, it is important to point out that the energy measurement can be avoided by modifying the detector design to provide four symmetric tubes parallel to the $z$-axis (see Fig. 3.3). As described in Refs. 4 and 5, for such a detector we obtain 


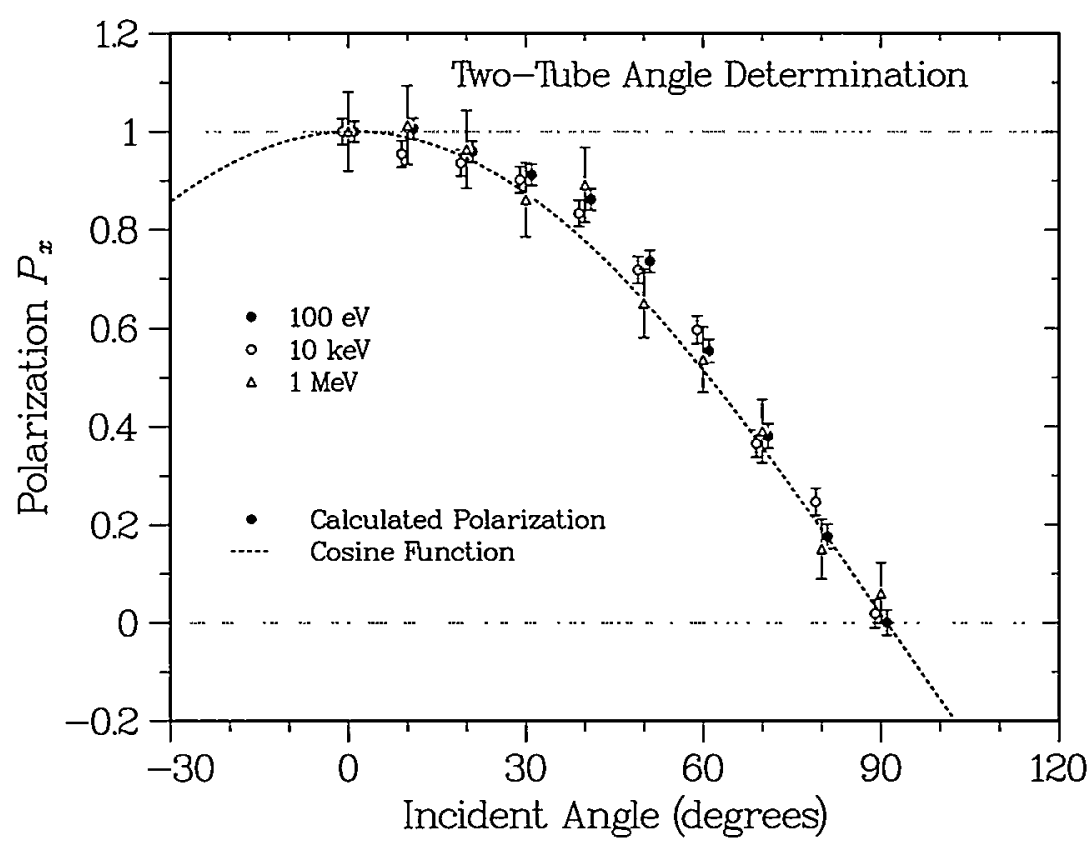

(a)

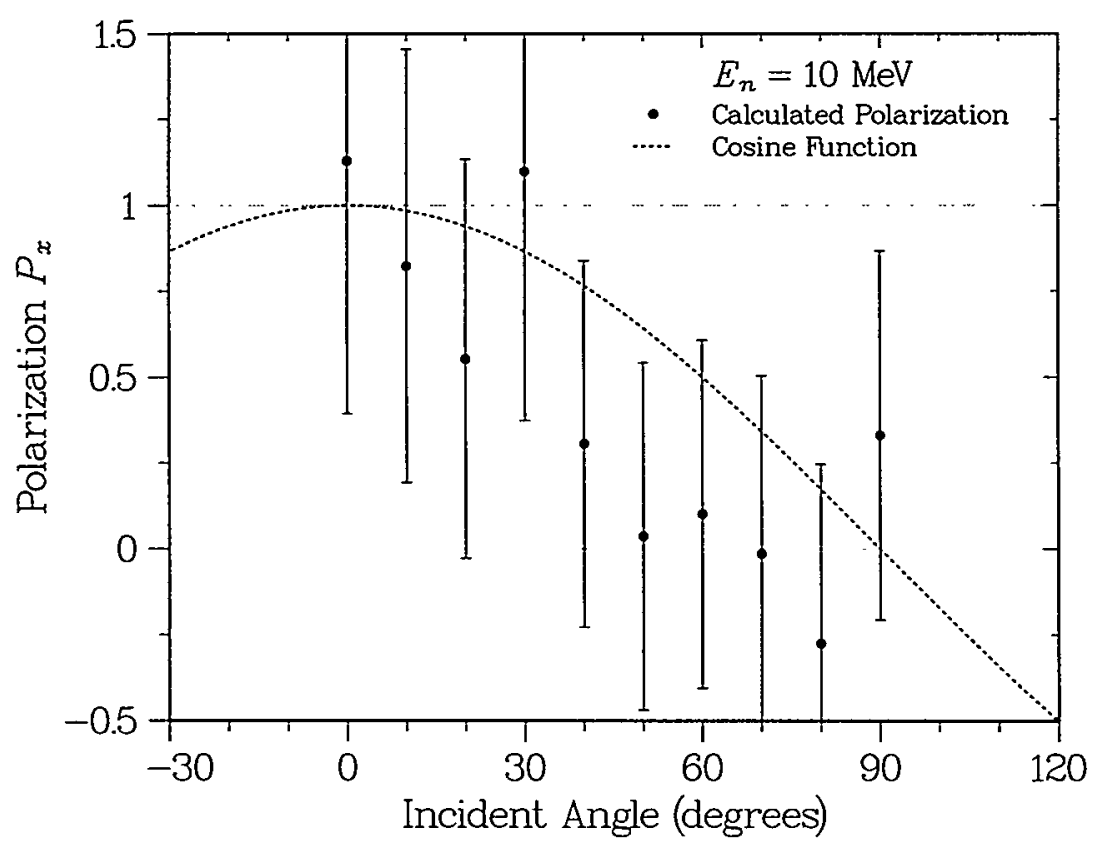

(b)

Figs. 3.2a,b. Two-tube angle determination. Converting the asymmetry in the measured two-tube count rates to a component of the incident polarization reveals a good correlation with the cosine of the incident angle. Part a shows the low-energy results; part $\mathrm{b}$ shows the $10-\mathrm{MeV}$ results, whose usefulness is severely limited by the near-zero analyzing power at high energies. 


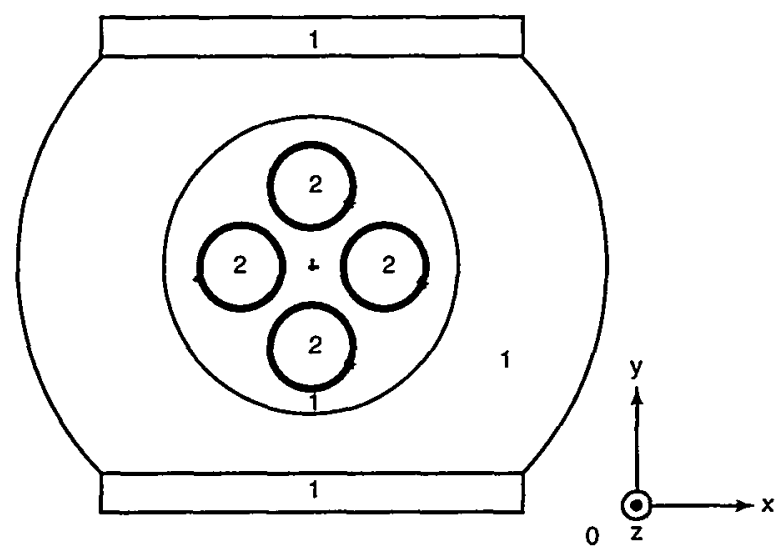

FRONT

Fig. 3.3. Computer model of a modified detector with four symmetrically placed ${ }^{3} \mathrm{He}$ tubes.

$$
\begin{gathered}
r_{x}=N_{+x} / N_{-x}, \quad r_{y}=N_{+y} / N_{-y} \\
R_{x}=\left(r_{x}-1\right) /\left(r_{x}+1\right), \quad R_{y}=\left(r_{y}-1\right) /\left(r_{y}+1\right) \\
R^{2}=\left(R_{x}^{2}+R_{y}^{2}\right) \\
u=R_{y} / R_{x}, \quad \theta=\tan ^{-1} u .
\end{gathered}
$$

The error analysis assumes that the fractional errors $\epsilon_{i}=d N_{i} / N_{i}$ are given by $\sqrt{1 / N_{i}}$, so that the corresponding $\epsilon_{x}$ and $\epsilon_{y}$ values for the two components $\left(R_{x}, R_{y}\right)$ are

$$
\begin{gathered}
d r_{x}=r_{x}\left(\epsilon_{+x}^{2}+\epsilon_{-x}^{2}\right)^{1 / 2}, \quad d r_{y}=r_{y}\left(\epsilon_{+y}^{2}+\epsilon_{-y}^{2}\right)^{1 / 2} \\
d R_{x}=2 /\left(r_{x}+1\right)^{2} d r_{x}, \quad d R_{y}=2 /\left(r_{y}+1\right)^{2} d r_{y} \\
\epsilon_{R}=\left(R_{x}^{2} d R_{x}^{2}+R_{y}^{2} d R_{y}^{2}\right)^{1 / 2} / R \\
d u=u\left(\epsilon_{x}^{2}+\epsilon_{y}^{2}\right)^{1 / 2} \\
d \theta=d u /\left(1+u^{2}\right) .
\end{gathered}
$$

The results of this approach for four tubes (again at separations of $\pm 2.0 \mathrm{~cm}$ ) are shown in Figs. 3.4a,b for low energies (part a) and at $10 \mathrm{MeV}$ (part b). Again, there is no recourse in the case of the zero analyzing power at the highest energies (part b), but in Fig. 3.4a the agreement between the actual and measured angles is within the statistical uncertainties, except for the small effect of inter-tube shielding evident in the $100-\mathrm{eV}$ distribution. 


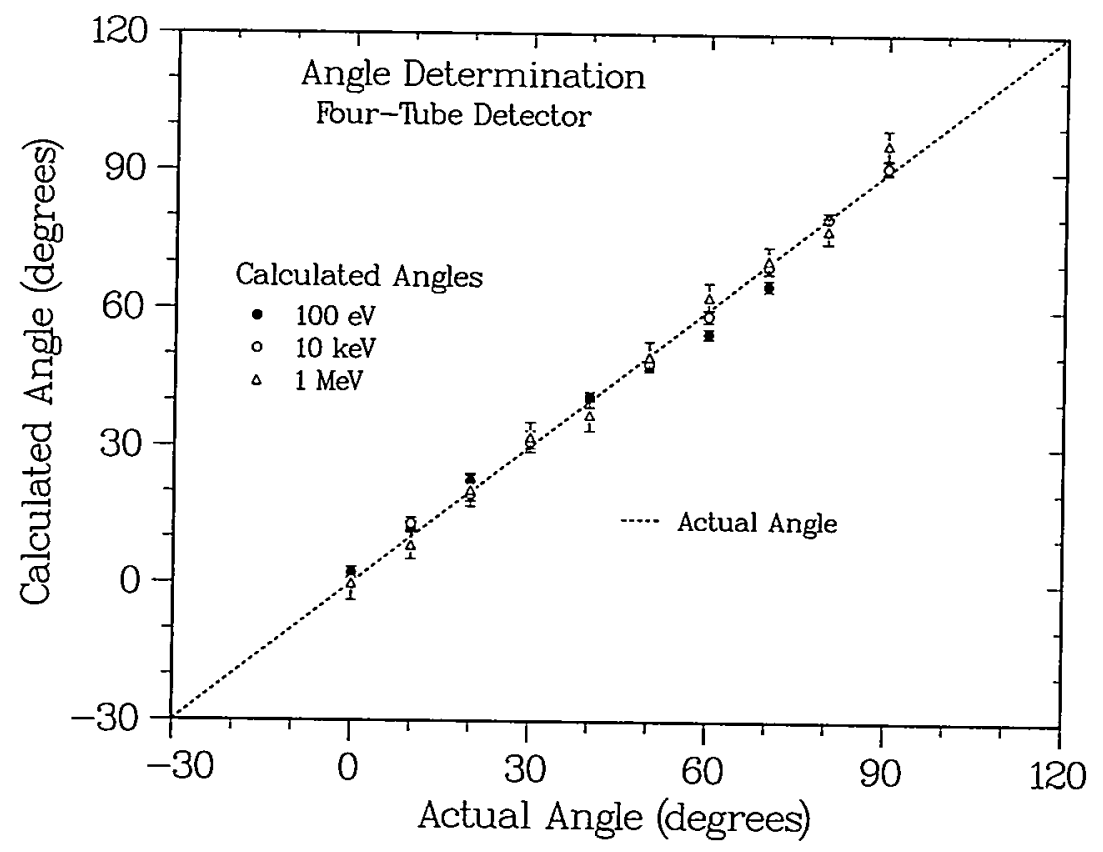

(a)

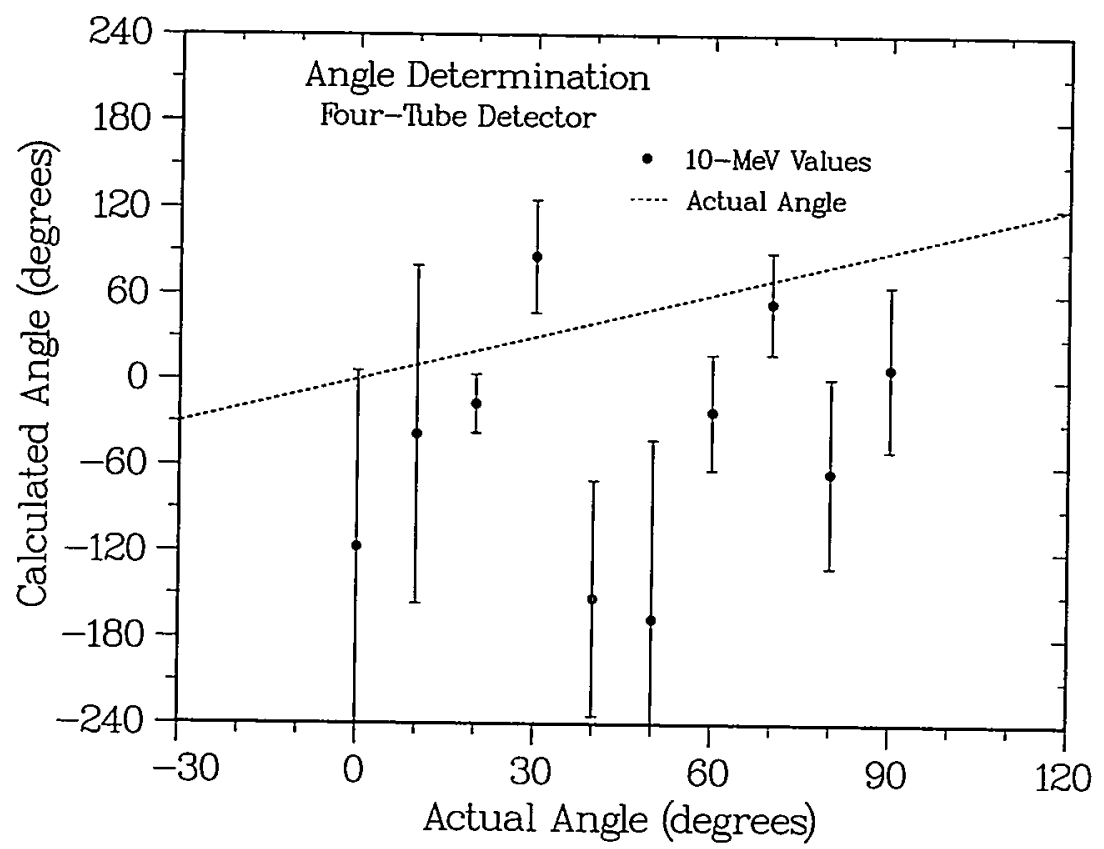

(b)

Figs. 3.4a,b. Four-tube angle determination. Installing four parallel counters in the moderator allows the $x$ and $y$ asymmetries to be measured simultaneously, which allows the incident angle to be determined without knowledge of the neutron energy. Part a shows the low-energy results; part b shows the $10-\mathrm{MeV}$ results. 
Summary. This section has exploited asymmetries in the count rates for a multi-tube detector to estimate the angle of incidence for the incoming neutron flux. This approach is similar to one developed for a four-rod scintillation detector, ${ }^{4,5}$ except that now the detector hardware is simpler and a two-tube formulation is also provided. Unfortunately, this twotube approach is limited to cases where the neutron energy can be measured or is known to be below about $0.1 \mathrm{MeV}$. Although not explored here, it is likely that orienting three tubes along the three orthogonal axes would provide an unambiguous signature for any direction; using pairs of tubes would probably also allow a general determination of the incident angle. It is also important to note the tradeoff between minimum detector size and good directionality (that is, high analyzing power). Obtaining maximum efficiency requires placing the tubes in an optimum region determined by balancing sufficient moderation against increasing attenuation. Obtaining a high analyzing power requires deliberately moving the tubes away from this optimum region to provide a difference in count rate. Therefore, only by placing the tubes at nonoptimum locations for both efficiency and directionality can one accurately measure both count rate and count-rate asymmetry simultaneously.

\section{IMPROVEMENTS IN HIGH-ENERGY DETECTION EFFICIENCY}

Motivation. Because the moderating effectiveness of the hydrogen in polyethylene follows the $n-p$ cross section, obtaining high detection efficiency at energies above $10 \mathrm{MeV}$ requires moderator sizes that are at odds with the need for lightweight instruments in spacebased applications. A similar situation arises for neutron dosimetry in the radiation fields around high-energy accelerators, where the decreasing response of instruments developed for lower-energy fission sources can seriously underestimate the biological effects of high-energy neutrons, and the increased weight of larger instruments limits their portability. Because it is very desirable to provide a dosimeter whose output scales directly with neutron dose, significant effort has been invested in designing detectors that approach this proportionality. A surprisingly successful technique simply adds a shell of lead shielding to the interior of the detector. ${ }^{7,8}$ Basically, the increase in $(\mathrm{n}, 2 \mathrm{n}$ ) neutron production above $10 \mathrm{MeV}$ provides some multiplication, and the large energy loss from the neutron-nucleus binding energy provides a convenient mechanism for lowering the mean neutron energy into the few-MeV range where a polyethylene moderator of modest size is most effective. It is reasonable to expect that such a modification would improve our detector's efficiency in the 10 - to 20 $\mathrm{MeV}$ range, but at an obvious cost of significantly increased overall mass-unless, of course, the increase in efficiency can be offset by a compensating reduction in moderator size. The search for this compromise is the subject of this section of the report. All calculations will use the rotated moderator, and all will use random incidence to average over different detector orientations.

Outer Lead Shell. The detector geometry used in the high-energy designs places a lead shell between layers of polyethylene at both smaller and larger radii. 7,8 Our moderator is comparable in size to the inner moderator in these dosimeters, and any energy loss in passing through an outer moderator would be expected to reduce the multiplication provided by the $(n, 2 n)$ reaction. Thus, our initial tests compare the effective area for our standard 9.144$\mathrm{cm}$ polyethylene moderator with that for the same detector surrounded by a $0.3-\mathrm{cm}$ lead shell. The results are shown in Figs. 4.1a,b. As hoped, (1) there is essentially no effect on the response below about $100 \mathrm{keV},(2)$ there is only a slight decrease in the $1-\mathrm{MeV}$ peak efficiency, and (3) there is actually a small increase in the effective area at $10-20 \mathrm{MeV}$ (see part b). The size of this increase, about $10 \%$ at $8 \mathrm{MeV}$ and $25 \%$ at $14 \mathrm{MeV}$, is consistent with that reported in other studies. ${ }^{7,8}$ However, when scaled as the estimated effective area per unit mass, as shown in Fig. 4.1c, the results are clearly unfavorable at all energies, with a $50 \%$ loss at $14 \mathrm{MeV}$. 


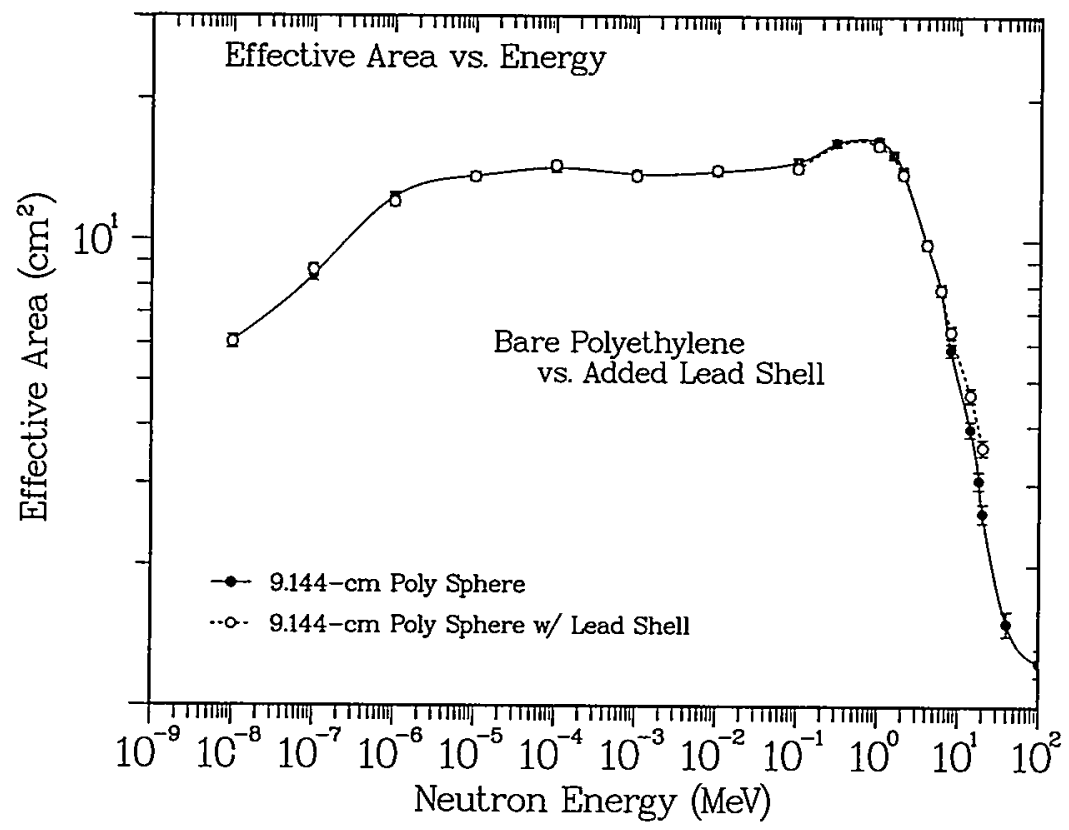

(a)

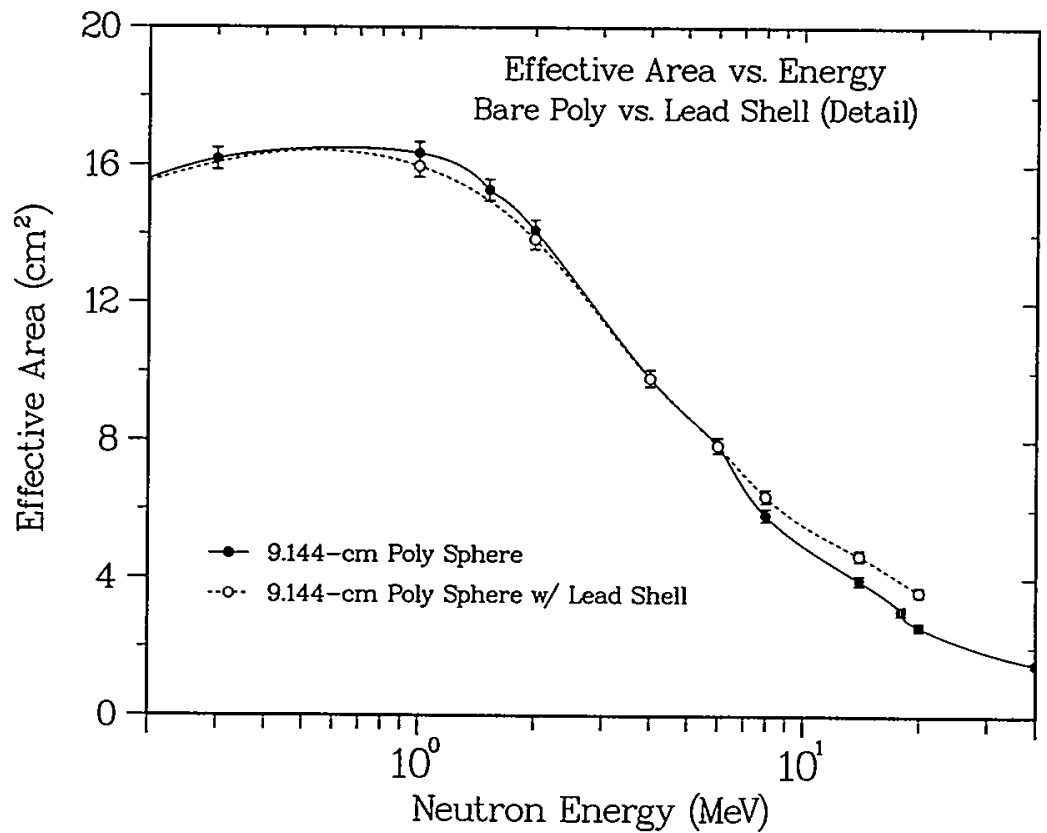

(b)

Figs. 4.1a,b. Effective area for a detector with a $0.3-\mathrm{cm}$ lead shell surrounding our standard $9.144-\mathrm{cm}$ polyethylene moderator. Part a shows the full energy range; part $b$ is an expanded view of the high-energy region. 


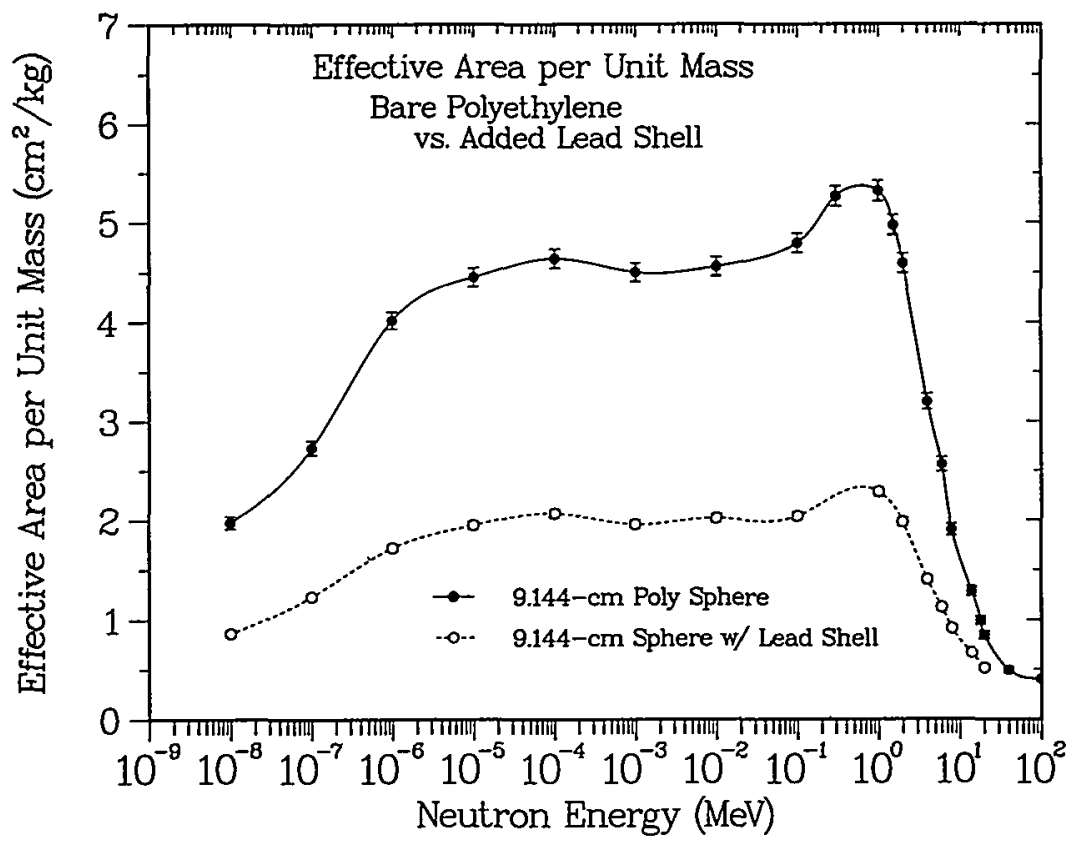

Fig. 4.1c. Effective areas as in Fig. 4.1a but divided by the estimated total detector mass. The added lead shell reduces the effectiveness of the detector by more than $50 \%$.

Lead Shells and Smaller Moderators. In an attempt to recover the effective area per unit mass of the standard detector, we have reduced the moderator radius by the $\sim 5 \%$ value used for Fig. $2.3 \mathrm{~b}$ and again added a surrounding lead shell. The hope is that the multiplication from the $(n, 2 n)$ reaction will compensate for the efficiency loss caused by the decreased moderation; optimistically, a large effect might even compensate for the added mass of the lead. The results are shown in Figs. 4.2a,b. Although the high-energy efficiencies of the two spheres are about the same (part a), the equivalence disappears completely when plotted on a per-mass basis (part b).

Uniform Loading. As an alternate approach, we next explore the effect of adding different materials uniformly throughout the polyethylene moderator. Preliminary studies suggested that $30 \%$ loading by weight provided a reasonable compromise between increased weight and any improvement in high-energy efficiency. In addition to loading with lead, we also investigated uniform loading with $30 \%$ tungsten (because of its high density and the ready availability of tungsten carbide) and with $30 \%$ beryllium (because of its low density and high $(n, 2 n)$ cross section for fast neutrons). These calculations are shown in Figs. 4.3a,b. First, the lead-loading results (part a) demonstrate that there is a slight loss at high energies $(\sim 30 \%$ at $14 \mathrm{MeV})$ and little effect below $10 \mathrm{MeV}$, where the replacement of polyethylene with lead results in a decrease in attenuation. Second, part b shows that, even without considering mass, tungsten causes losses at all energies because of its low-energy neutron absorption. However, it appears that for beryllium the low density and high $(n, 2 n)$ yield do provide a small, but significant, improvement at most energies (including a 30\% gain in area at $14 \mathrm{MeV}$ ), although at the cost of working with a very hazardous substance. As a final test, in Figs. 4.4a,b we compare the effective areas per unit mass for moderators using standard polyethylene, lead loading, and beryllium loading. Although the lead case is a clear disadvantage, the beryllium loading actually improves the efficiency per unit mass for energies above about $10 \mathrm{MeV}$, with an $11 \%$ improvement at $14 \mathrm{MeV}$. However, this 


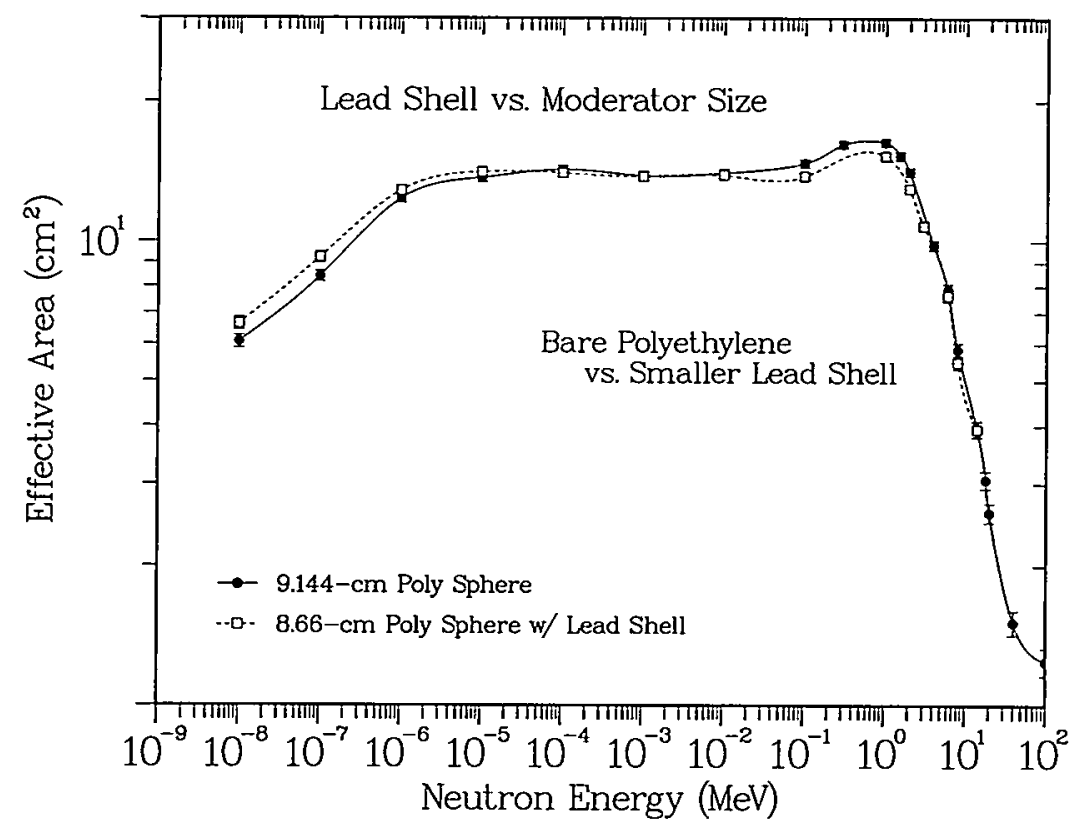

(a)

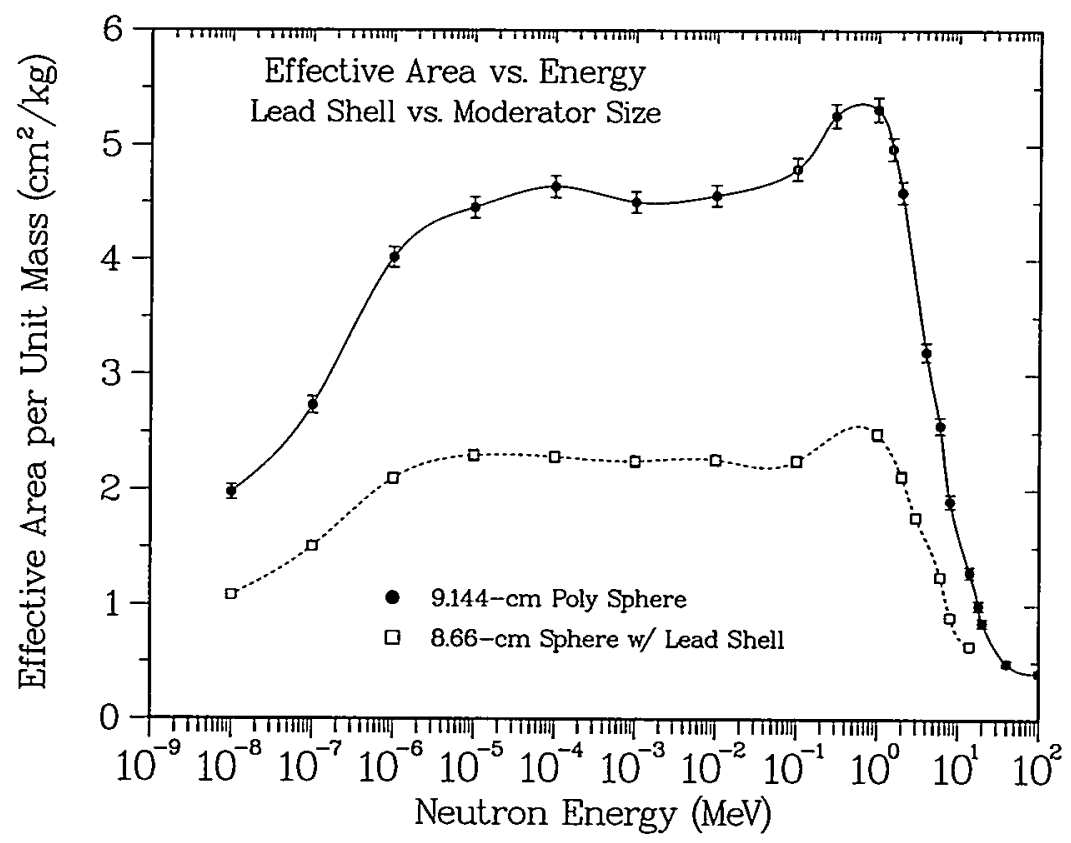

(b)

Figs. 4.2a,b. Effective areas for the standard 9.144-cm sphere (without added lead) and a smaller $8.66-\mathrm{cm}$ sphere surrounded with a $0.3-\mathrm{cm}$ lead shell. In part a, at high energies the (n,2n) neutron production approximately cancels out the efficiency loss from the smaller moderator (compare with Fig. 2.3b). Part b shows the normalization to total mass, which demonstrates that the compromise still results in much lower efficiency per unit mass. 


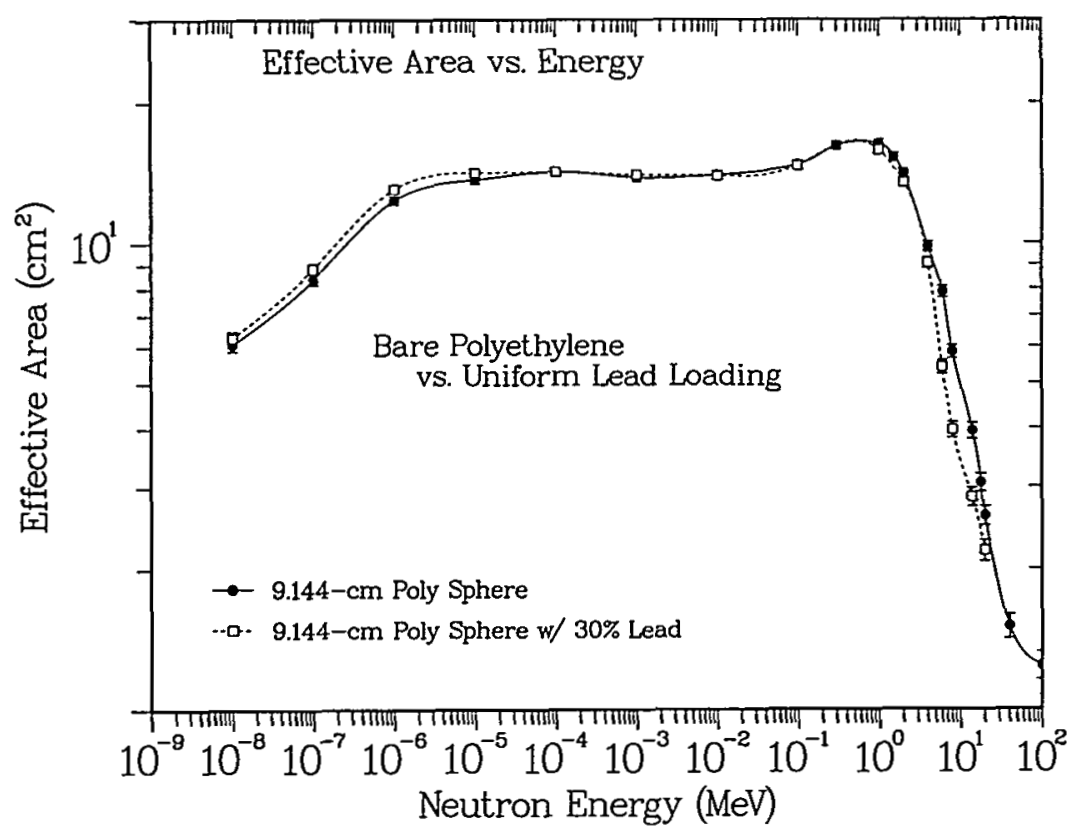

(a)

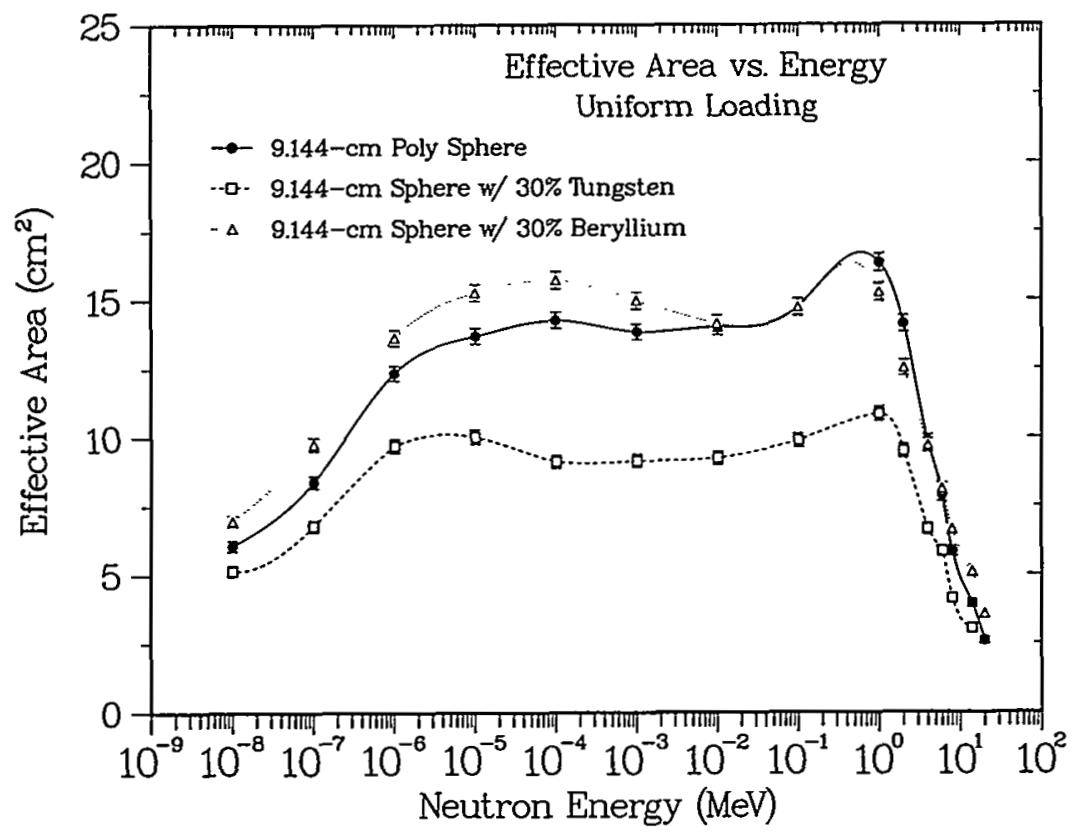

(b)

Figs. 4.3a,b. Part a: Effective areas for the standard polyethylene sphere and a sphere of the same size but made of polyethylene loaded with $30 \%$ lead by weight. The added lead has essentially the same effect as decreasing the amount of moderation (compare with Fig. 2.3a). Part b: As in part a, but for loading with tungsten and beryllium. Adding tungsten lowers the efficiency at all energies; adding beryllium actually raises the efficiency in many cases. 


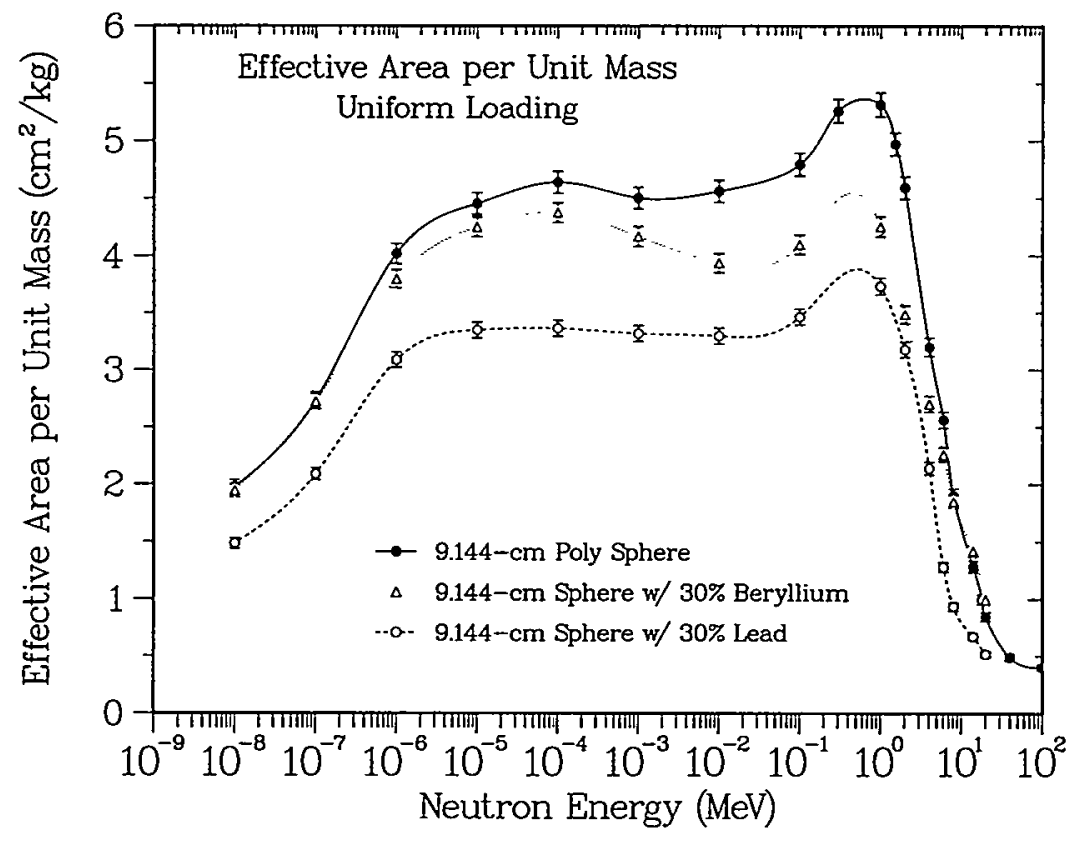

(a)

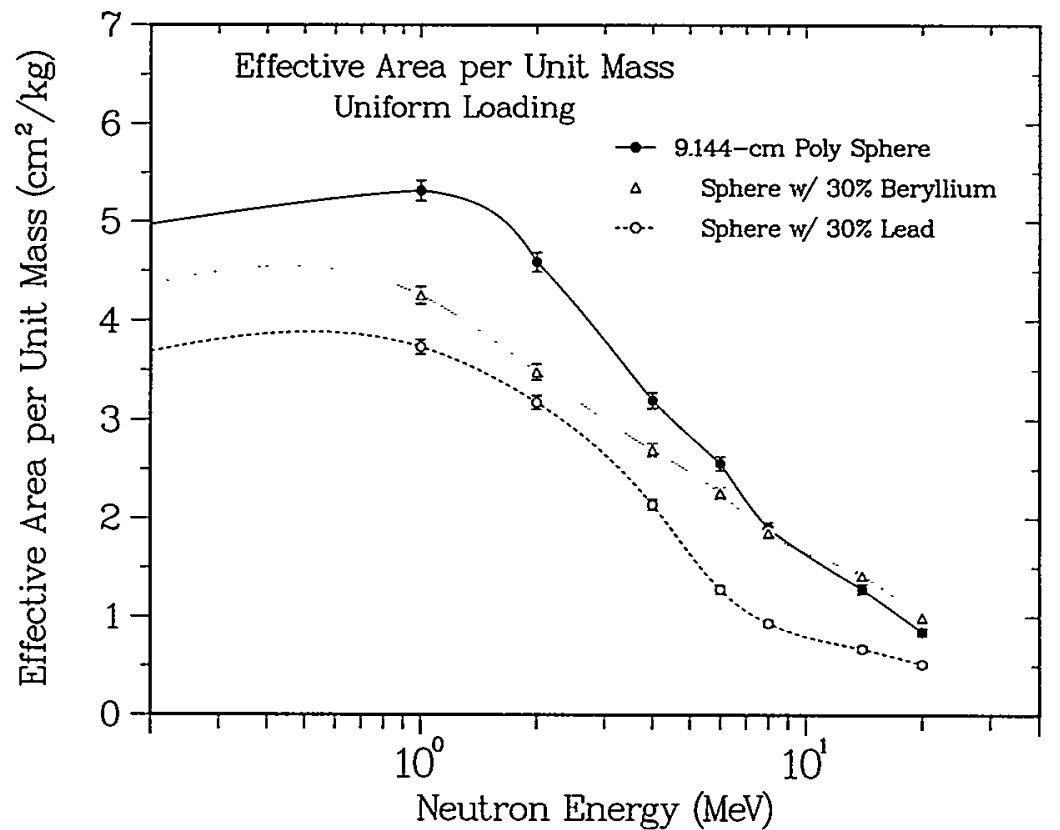

(b)

Figs. 4.4a,b. Effective areas per unit mass for uniform loading with different materials. Part a shows the full energy range; part $b$ focuses on the region at the highest energies, where adding beryllium improves the efficiency per unit mass very slightly. 
improvement is more than counterbalanced by the detector's decreased effectiveness at all lower energies.

Conclusions. For the addition of $0.3 \mathrm{~cm}$ of lead to the outside of our 9.144-cm polyethylene sphere, the $\sim 25 \%$ improvement in efficiency at $14 \mathrm{MeV}$ (see Fig. $4.1 \mathrm{~b}$ ) is similar to the results reported elsewhere. ${ }^{7,8}$ On a per-mass basis, however, the addition of lead is clearly not effective at our energies and with our detector size. It is certain that much greater improvements can be obtained at higher energies, because the (n,2n) production rises rapidly, while $n-p$ scattering drops rapidly to the point that carbon breakup reactions become a more effective mechanism for neutron moderation. At very high energies it may even be possible that a lead-loaded detector has a higher efficiency per unit mass than a standard polyethylene instrument with the same effective area. For energies below $20 \mathrm{MeV}$, however, we have achieved such success only with the addition of beryllium, and even then the improvement per unit mass is almost negligible and exists only at energies above $10 \mathrm{MeV}$. It is, however, interesting to compare our negative results with those obtained for the case of the boron- or tungsten-loaded moderators that have been proposed for neutron dosimetry. These strongly absorbing materials are employed to deliberately tailor the detector's sub-MeV response to match the desired energy-dose relations. ${ }^{7}$ In such cases the addition of lead can in fact cause the high-energy efficiency to increase, simply because the lead replaces some of the neutron-absorbing material. Careful consideration of detector geometry is needed to optimize such response.

\section{SUMMARY AND RELATED WORK}

Effective Area versus Efficiency. As developed previously for scintillation detectors, ${ }^{6}$ replacing the conventional efficiency per incident neutron with efficiency per unit fluence, that is, effective detector area, avoids difficulties with irregular detector geometries or angular distributions. For the polyethylene-moderated ${ }^{3} \mathrm{He}$ tubes used in space-based neutron detectors, ${ }^{3}$ this replacement allows us to easily determine detailed response characteristics as functions of both energy and angle, in the process correcting some mistakes in the earlier report.

Directionality. By exploiting the different efficiencies of multiple-tube detectors for neutrons from different directions, we can use differences between counting rates to provide information about the direction of the incident neutrons. Our development in terms of a detector analyzing power emphasizes the importance of placing the tubes to provide a deliberate asymmetry in their counting rates, which necessarily comes at some loss in detection efficiency. Four-tube detectors can provide accurate angle information about two axes; two-tube approaches can provide similar information but require a separate determination of the incident neutron energy, such as by timing the neutrons' arrival relative to their production. For such pulsed sources the temporal variation in the count-rate asymmetry provides an unmistakable signature of the detection of an incident neutron burst.

Efficiency Improvements. Although recent health-physics papers ${ }^{7,8}$ report improvements in high-energy efficiency by adding high-density materials to polyethylene moderators, for energies below $20 \mathrm{MeV}$ it seems that such improvements usually come at the cost of greatly increased detector mass. At our energies only the addition of low-density elements such as beryllium appear to provide even a negligible improvement on a per-mass basis. We therefore do not expect that such an approach will be useful for space-based detectors. 


\section{ACKNOWLEDGMENTS}

John Drabanski, a science teacher at Pecos High School in Pecos, New Mexico, spent the summer working in LANL's Nonproliferation and International Security Division. This report includes the results of his research using computer modeling, which he can further apply as he works with teachers from Russia, Mexico, and throughout the United States to help students better understand issues in science and technology.

This work was supported by the Research and Technology Division of the U.S. Department of Energy's Office of Nonproliferation and National Security. John Hendricks of the Radiation Transport Group provided a copy of the MCNP4A program. Richard Olsher located the references describing the LANL neutron calibration facility, and significant discussions of the contents were provided by Hsiao-Hua Hsu and William Casson. Michael Meier provided a critical reading of the final manuscript. 


\section{REFERENCES}

1. S. J. Bame, J. P. Conner, F. B. Brumley, R. L. Hostetler, and A. C. Green, "Neutron Flux and Energy Spectrum above the Atmosphere," Journal of Geophysical Research 68, 1221-1228 (1963).

2. R. L. Bramblett, R. I. Ewing, and T. W. Bonner, "A New Type of Neutron Spectrometer,"Nuclear Instruments and Methods 9, 1-12 (1960).

3. R. C. Byrd, B. A. Barraclough, W. C. Feldman, and S. J. Bame, "Measurements and Calculations of the Characteristics of a Space-Based Neutron Sensor," Los Alamos National Laboratory report LA-12340-MS (October 1992).

4. R. C. Byrd, "Directional Neutron Detectors," Los Alamos National Laboratory report LA-12379-MS (October 1992).

5. Roger Byrd, George Auchampaugh, and William Feldman, "Directional Measurements for Sources of Fission Neutrons," Los Alamos National Laboratory report LA-12633MS (November 1993).

6. R. C. Byrd and W. T. Urban, "Calculations of the Neutron Response of BoronLoaded Scintillators," Los Alamos National Laboratory report LA-12833-MS (December 1994).

7. C. Biratti, A. Esposito, A. Ferrari, M. Pelliccioni, and M. Salari, "Calibration of the Neutron Rem Counter LINUS in the Energy Range from Thermal to $19 \mathrm{MeV}$," Nuclear Instruments and Methods A324, 232-238 (1993).

8. H. H. Hsu, K. R. Alvar, and D. G. Vasilik, "A New Bonner-Sphere Set for High Energy Neutron Measurements: A Monte Carlo Simulation," IEEE Transactions on Nuclear Science NS-41, 938-940 (1994).

9. J. F. Briesmeister, Ed., "MCNP-A General Monte Carlo N-Particle Transport Code, Version 4A," Los Alamos National Laboratory report LA-12625-M (November 1993).

10. Richard H. Olsher, "Calibration Correction Factors for the Los Alamos Neutron Well," Los Alamos National Laboratory report LA-12127-MS (August 1991).

11. W. F. Harvey and F. Hajnal, "Multisphere Neutron Spectroscopy Measurements at the Los Alamos National Laboratory Plutonium Facility," Radiation Protection Dosimetry 50, 13-30 (1993). 
Appendix A. MCNP Input Deck

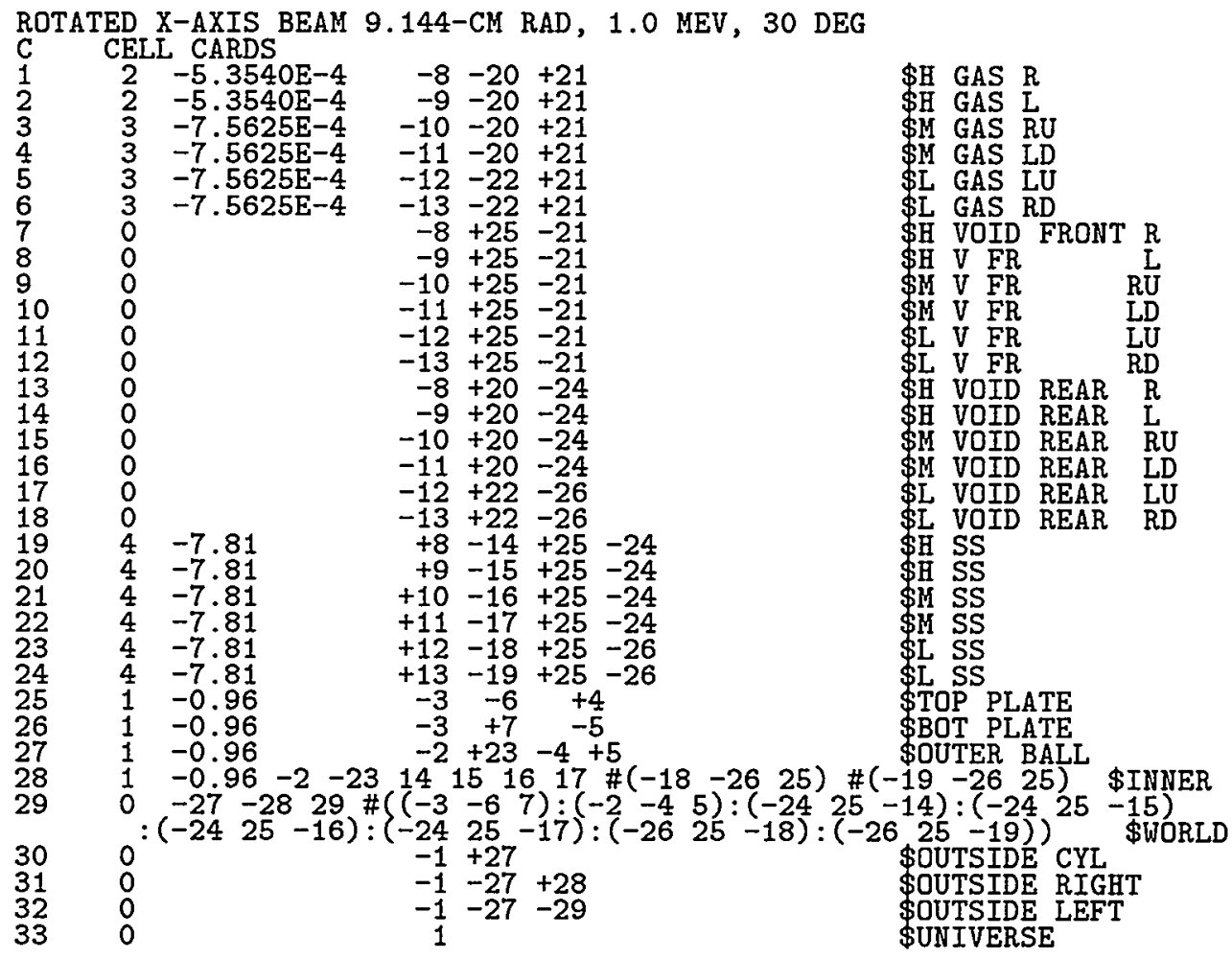

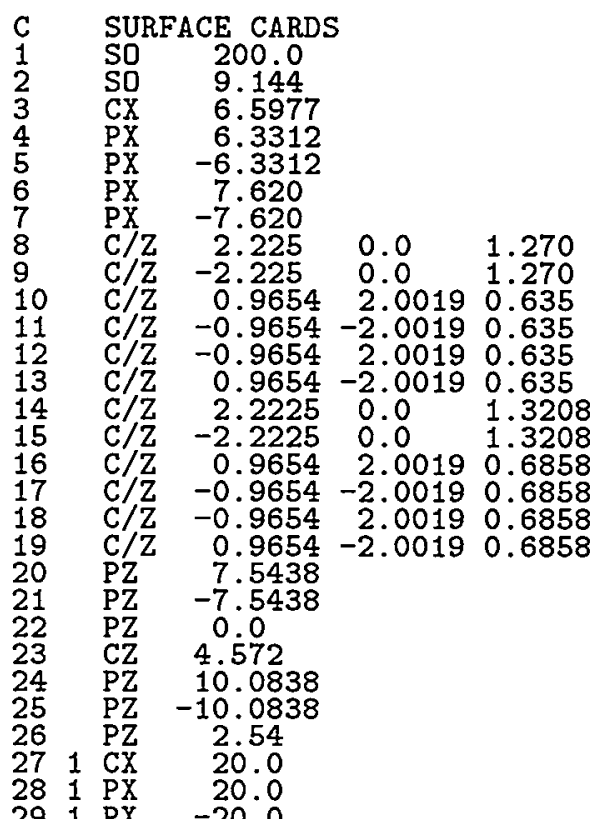

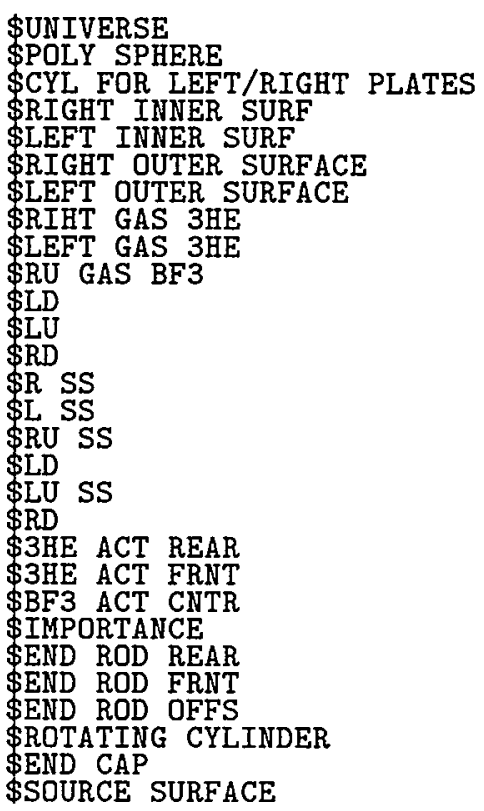

C DATA CARDS

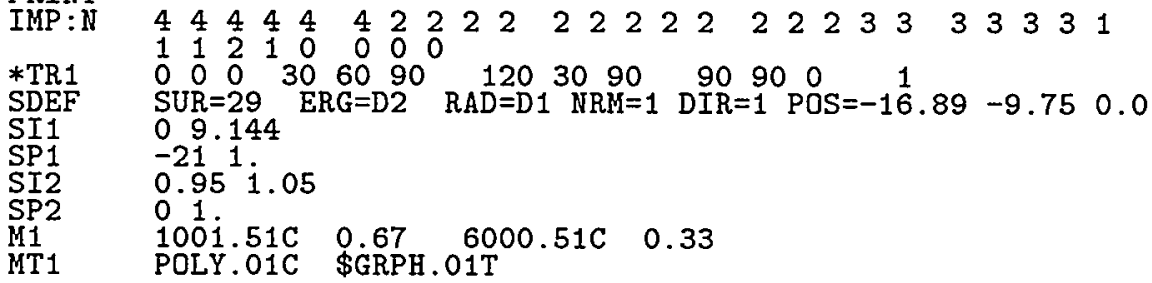




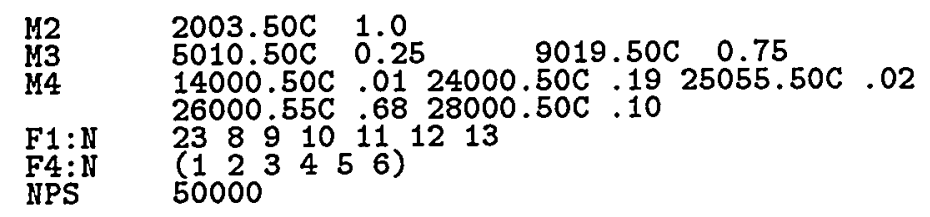

As noted in the text, in the actual detector the mounting surfaces are rotated by $90^{\circ}$ from the orientation shown above. The corrected versions of surfaces 3-7 (with the $x$ - and $y$-axes changed) follows:

$\begin{array}{llr}3 & \text { CY } & 6.5977 \\ 4 & \text { PY } & 6.3312 \\ 5 & \text { PY } & -6.3312 \\ 6 & \text { PY } & 7.620 \\ 7 & \text { PY } & -7.620\end{array}$

\$CYL FOR TOP/BOTTOM PLATES

\$TOP-INHER SURF

\$BOT-INNER SURF

\$BOT-OUTER SURF 


\section{Appendix B. Actual Detector Responses}

For future reference, we include here the numerical values for the effective areas (Fig. 2.6) and $x$-axis analyzing powers (Fig. 3.1 ) for the actual detector.

\begin{tabular}{crrcc}
$\begin{array}{c}\text { Energy } \\
(\mathrm{MeV})\end{array}$ & $\begin{array}{c}A_{\text {eff }} x \text {-axis } \\
\left(\mathrm{cm}^{2}\right)\end{array}$ & $\begin{array}{c}A_{\text {eff }} y \text {-axis } \\
\left(\mathrm{cm}^{2}\right)\end{array}$ & $\begin{array}{c}A_{\text {eff }} z \text {-axis } \\
\left(\mathrm{cm}^{2}\right)\end{array}$ & $\begin{array}{c}\text { Analyzing } \\
\text { Power }(\%)\end{array}$ \\
\hline $10^{-8}$ & $2.87 \pm 0.02$ & $1.81 \pm 0.02$ & $15.89 \pm 0.26$ & 84.4 \\
$10^{-7}$ & $5.10 \pm 0.04$ & $4.03 \pm 0.07$ & $19.22 \pm 0.28$ & 80.9 \\
$10^{-6}$ & $9.10 \pm 0.11$ & $7.24 \pm 0.10$ & $21.30 \pm 0.29$ & 75.6 \\
$10^{-5}$ & $10.94 \pm 0.13$ & $9.61 \pm 0.12$ & $18.67 \pm 0.26$ & 74.2 \\
$10^{-4}$ & $12.17 \pm 0.14$ & $10.37 \pm 0.12$ & $15.94 \pm 0.23$ & 68.8 \\
$10^{-3}$ & $12.28 \pm 0.14$ & $11.03 \pm 0.13$ & $14.62 \pm 0.22$ & 67.8 \\
$10^{-2}$ & $12.32 \pm 0.14$ & $11.66 \pm 0.13$ & $13.65 \pm 0.21$ & 63.3 \\
$10^{-1}$ & $13.56 \pm 0.15$ & $13.51 \pm 0.15$ & $13.34 \pm 0.21$ & 54.1 \\
0.3 & $14.94 \pm 0.16$ & $15.92 \pm 0.16$ & $13.90 \pm 0.21$ & 46.7 \\
1.0 & $15.23 \pm 0.07$ & $15.86 \pm 0.16$ & $13.81 \pm 0.21$ & 29.0 \\
1.5 & $14.09 \pm 0.11$ & $15.02 \pm 0.16$ & $12.76 \pm 0.20$ & 25.0 \\
2 & $13.33 \pm 0.11$ & $14.41 \pm 0.11$ & $12.09 \pm 0.20$ & 17.4 \\
4 & $9.29 \pm 0.06$ & $10.23 \pm 0.08$ & & 13.9 \\
8 & $5.84 \pm 0.04$ & $6.13 \pm 0.05$ & $5.65 \pm 0.13$ & 5.4 \\
14 & $3.61 \pm 0.03$ & $3.78 \pm 0.03$ & & 2.0 \\
16 & & & $3.23 \pm 0.10$ & \\
20 & $2.65 \pm 0.02$ & $2.68 \pm 0.02$ & $2.34 \pm 0.08$ & 2.6
\end{tabular}

